\title{
Unsteady aspects of an incident shock wave/turbulent boundary layer interaction
}

\author{
R. A. HUMBLE广, F. SCARANO \\ AND B. W. VAN OUDHEUSDEN \\ Faculty of Aerospace Engineering, Delft University of Technology, \\ Kluyverweg 1, 2629 HS, Delft, The Netherlands
}

(Received 19 March 2008; revised 3 April 2009; accepted 6 April 2009)

An incident shock wave/turbulent boundary layer interaction at Mach 2.1 is investigated using particle image velocimetry in combination with data processing using the proper orthogonal decomposition, to obtain an instantaneous and statistical description of the unsteady flow organization. The global structure of the interaction is observed to vary considerably in time. Although reversed flow is often measured instantaneously, on average no reversed flow is observed. On an instantaneous basis, the interaction exhibits a multi-layered structure, characterized by a relatively highvelocity outer region and low-velocity inner region. Discrete vortical structures are prevalent along their interface, which create an intermittent fluid exchange as they propagate downstream. A statistical analysis suggests that the instantaneous fullness of the incoming boundary layer velocity profile is (weakly) correlated with the size of the separation bubble and position of the reflected shock wave. The eigenmodes show an energetic association between velocity fluctuations within the incoming boundary layer, separated flow region and across the reflected shock wave, and portray subspace features that represent the phenomenology observed within the instantaneous realizations.

\section{Introduction}

For more than half a century, the interaction between a shock wave and turbulent boundary layer (SWTBLI) has been one of the most outstanding challenges in highspeed fluid dynamics, and many critical questions still remain unanswered (Dolling 2001). In recent decades, there has been an evolving quest for a deeper understanding of the large-scale unsteadiness of SWTBLIs, in view of the apparent discrepancy in the time scale of the low-frequency motion of the shock system and separated flow region being of the order $10 \delta / U_{\infty}-100 \delta / U_{\infty}$, in contrast to the characteristic time scale of the incoming boundary layer $\delta / U_{\infty}$, where $\delta$ is the boundary layer thickness and $U_{\infty}$ is the free-stream velocity (see e.g. Dussauge, Dupont $\&$ Debiève 2006). While the small-scale motion has been typically attributed to the passage of turbulent structures within the incoming boundary layer, identifying the cause(s) of the large-scale motion has proven to be more problematic, and a cocktail of alternative hypotheses have been proposed.

Some of the most established and well-documented explanations for the large-scale unsteadiness include mechanisms involving the incoming boundary layer turbulence 
(see e.g. Andreopoulos \& Muck 1987), the internal dynamics of the separated flow region (see e.g. Dolling \& Murphy 1983; Erengil \& Dolling 1993), the incoming boundary layer velocity profile (Beresh, Clemens \& Dolling 2002) and large-scale coherent motions within the incoming boundary layer, in the form of streamwiseelongated low- and high-speed fluid (see Ganapathisubramani, Clemens \& Dolling 2007). Although not as well-documented as the compression ramp interaction investigations mentioned above, similar large-scale unsteadiness has also been found in the shock reflection case, such as the low-frequency motion of the reflected shock wave when boundary layer separation is involved.

For example Pirozzoli \& Grasso (2006) conducted a direct numerical simulation (DNS) of an incident SWTBLI and formulated a model based on an acoustic feedback mechanism, similar to what occurs in the generation of tones in cavities and screeching jets. Related experimental work has been analysed and reviewed by Dussauge et al. (2006), who suggested that the three-dimensional structure of the separation bubble may be at the origin of the large-scale unsteadiness. In particular, Dupont, Haddad \& Debiève (2006) obtained characteristic time and length scales of the unsteady reflected shock wave and inside the downstream interaction and found strong statistical evidence of a link between low-frequency shock movements and the downstream interaction. Yet to date, the large-scale unsteadiness still lacks a complete phenomenological exposition, and its precise cause(s) still remain a mystery.

The complexity of SWTBLIs therefore makes it desirable to distil from the evidence a simplified description of the overall dynamics that can be more readily understood. To achieve this, it is desirable to record accurate data that reveal the instantaneous structure of the flow field, and then seek an approach for the analysis and synthesis of the data that leads to the development of a low-order description while at the same time ensuring that the most important features are retained. One technique that has become popular in this role is the proper orthogonal decomposition (POD) (see Berkooz, Holmes \& Lumley 1993). In essence, the POD decomposes a signal into a basis of non-specified functions chosen to represent the energy of that signal in the fewest number of modes. These modes are constructed out of the spatial organization of the system in the most efficient manner, in the sense that a finite number of these modes represent more energy than any other set of orthogonal modes.

The technique also goes by other names, such as Karhunen-Loève decomposition, principal component analysis and the Hotelling transformation, and has been introduced into fluid dynamics by Lumley (1967) as a technique to highlight coherent motions within turbulent flows. It has been applied to a variety of fluid dynamic problems of practical interest, including boundary layers (Aubry et al. 1988) and compressible flows (Moreno et al. 2004), to produce low-dimensional representations of the flow phenomena present. Particle image velocimetry (PIV) data are particularly suited for the POD analyses, since the entire spatial velocity field is available by snapshots, leading to the construction of global eigenmodes that can be used to further characterize the instantaneous spatial organization of the flow. Its application to the SWTBLI problem is therefore considered to be well posed.

In the present paper, the unsteadiness of an incident SWTBLI at Mach 2.1 is investigated. The basic flow features are first outlined using PIV as the flow diagnostic technique. The interaction's instantaneous flow structure is then discussed, followed by a statistical analysis, to establish the characteristic features of the boundary layer, separated flow region and reflected shock wave, as elements in the large-scale unsteadiness. The flow field is then decomposed into a series of modes that capture the most dominant components of the unsteadiness using the POD. A simplified 


$\begin{array}{lc}\text { Parameter } & \text { Quantity } \\ M_{\infty} & 2.1 \\ U_{\infty}\left(\mathrm{m} \mathrm{s}^{-1}\right) & 518 \\ \delta_{99}(\mathrm{~mm}) & 20 \\ C_{f} & 1.52 \times 10^{-3} \\ P_{0}(\mathrm{kPa}) & 276 \\ T_{0}(\mathrm{~K}) & 286 \\ \operatorname{Re}_{\theta} & 4.92 \times 10^{4} \\ \operatorname{Re}_{\tau} & 8600\end{array}$

TABLE 1. Experimental conditions.

conceptual model of the interaction's unsteady flow organization is then formulated based on a consolidation of the results.

\section{Experimental facility and procedure}

Experiments were performed in the blow-down transonic-supersonic wind tunnel (TST-27) of the High-Speed Aerodynamics Laboratories at Delft University of Technology. The facility generates flows in the Mach number range 0.5-4.2. The Mach number can be set by means of a continuous variation of the throat section and flexible nozzle walls. The tunnel operates at unit Reynolds numbers typically ranging from $30 \times 10^{6}$ to $130 \times 10^{6} \mathrm{~m}^{-1}$, enabling an operating use of approximately $300 \mathrm{~s}$. In the present study, the test section had a height of $255 \mathrm{~mm}$ and a width of 280 $\mathrm{mm}$. The tunnel was operated at a nominal free-stream Mach number $M_{\infty}=2.1$ (free-stream velocity $U_{\infty}=518 \mathrm{~m} \mathrm{~s}^{-1}$ ), with a stagnation pressure of $P_{0}=276 \mathrm{kPa}$ and stagnation temperature of $T_{0}=286 \mathrm{~K}$. Hot-wire anemometry measurements performed in the free-stream of the test section determined the turbulence intensity to be approximately $1 \%$ of $U_{\infty}$.

The boundary layer developing along the floor of the wind tunnel was investigated in the present experiment. The boundary layer developed along a smooth surface under nearly adiabatic flow conditions for a development length of approximately $2 \mathrm{~m}$. On entering the measurement domain, the boundary layer thickness $(99 \%$ of $U_{\infty}$ ) was $\delta=20 \mathrm{~mm}$, with displacement thickness $\delta^{*}=4.4 \mathrm{~mm}$ and momentum thickness $\theta=1.4 \mathrm{~mm}$. A log-law least squares fit in combination with the van Driest transformation was used to determine the skin friction coefficient $C_{f}=1.52 \times 10^{-3}$ corresponding to a friction velocity $u_{\tau}=\sqrt{\left(\tau_{w} / \rho_{w}\right)}=19.4 \mathrm{~m} \mathrm{~s}^{-1}$. The Reynolds number based on the momentum thickness is denoted by $\operatorname{Re}_{\theta}=U_{\infty} \theta / v_{\infty}=4.92 \times 10^{4}$ (where $v_{\infty}$ is the kinematic viscosity in the free-stream). The Reynolds number based on the friction velocity and boundary layer thickness is denoted by $R e_{\tau}=u_{\tau} \delta / v_{w}=8600$ (where $v_{w}$ is the kinematic viscosity at the wall). The experimental conditions are summarized in table 1. A statistical description of the interaction in terms of its mean flow pattern and turbulence properties has been reported in a previous work by Humble, Scarano \& van Oudheusden (2007) and will not be repeated here. However, the streamwise and wall-normal root mean square (r.m.s.) velocity components of the incoming boundary layer were found to agree well with the two-component PIV results of Hou (2003) and the hot-wire anemometry of Elena \& Lacharme (1988), for example (see also Humble et al. 2009 for a further comparison).

A shock generator was placed in the middle of the test section to generate the incident shock wave. The generator consisted of a $100 \mathrm{~mm}$ chord single-sided wedge 


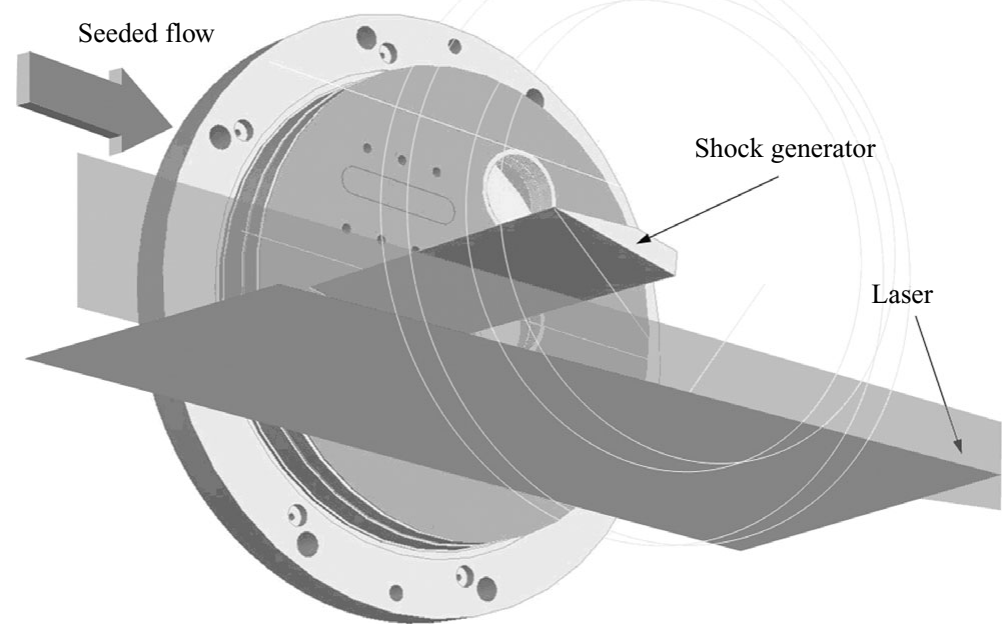

FIGURE 1. Schematic of the experimental arrangement. Laser illumination is from downstream of the shock generator. The flow direction is from left to right.

giving a flow deflection angle of $8^{\circ}$. The generator was rigidly mounted on one side of the wind tunnel and spanned $96 \%$ of the test section. Figure 1 shows a schematic of the experimental arrangement. A spanwise multi-planar PIV assessment by Humble et al. (2007) has demonstrated that the mean flow field remains largely symmetrical about the centreline, with the velocity components varying less than $5 \%$ within the spanwise region $-2.5 \leqslant z / \delta \leqslant 2.5$, where $z=0$ is the centreline of the test section. This has been recently substantiated by surface oil-flow visualization of a very similar interaction by Humble et al. (2009).

Two-component PIV was employed in the present study. A two-dimensional rake seeded the flow in the settling chamber with UV-TITAN titanium dioxide $\left(\mathrm{TiO}_{2}\right)$ as tracer particles, with a manufacturer-specified nominal diameter of $50 \mathrm{~nm}$ and a bulk density of $200 \mathrm{~kg} \mathrm{~m}^{-3}$. The particle relaxation time across the incident shock wave has been previously estimated to be $\tau_{p} \approx 2.1 \mu \mathrm{s}$, corresponding to a frequency response $f_{p} \sim 450 \mathrm{kHz}$ (see Humble et al. 2007). The effective (agglomerated) diameter of the particles has been estimated from electron microscopy scans of the porous agglomerates and found to be $d_{p} \sim 400 \mathrm{~nm}$ (see Schrijer, Scarano \& van Oudheusden 2006). As suggested by Samimy \& Lele (1991), the particle dynamic effects may be further parameterized by the Stokes number $S t=\tau_{p} / \tau_{f}$ (where $\tau_{f}$ is the flow time scale of interest). For accurate flow tracking at the time scale of $\tau_{f}$, it is necessary to meet the criterion that $S t \ll 1$. The present Stokes number, taking $\tau_{f}$ to be the characteristic outer flow time scale $\delta / U_{\infty} \approx 40 \mu \mathrm{s}$, is about 0.06 , indicating that the particles track the flow with fidelity at the time scale of $\tau_{f}$.

Flow illumination was provided by a Big Sky CFR PIV-200 Nd:Yag laser with $200 \mathrm{~mJ}$ pulsed energy and a $7 \mathrm{~ns}$ pulse duration at wavelength $532 \mathrm{~nm}$. Laser light access was provided by a probe inserted into the flow downstream of the shock generator. The laser pulse separation was $2 \mu \mathrm{s}$, resulting in a particle displacement of $1 \mathrm{~mm}$ in the free-stream flow. The light sheet was positioned in the middle of the test section and was approximately $1.5 \mathrm{~mm}$ thick. Images were recorded by a PCO Sensicam QE, a 12-bit charged-couple device camera with a sensor of size $1376 \times 1040$ 
Parameter

Field of view

Interrogation volume

Digital image resolution

Objective focal length

$f$-number

Laser pulse separation
Quantity

$129 \mathrm{~mm}$ length, $40 \mathrm{~mm}$ height

$1.9 \times 1.6 \times 1.5 \mathrm{~mm}^{3}$

$\approx 11$ pixels $\mathrm{mm}^{-1}$

$f=60 \mathrm{~mm}$

$f_{\#}=8$

$2 \mu \mathrm{s}$

TABle 2. Summary of PIV recording parameters.

pixels. Only 416 pixel rows were used given the large aspect ratio of the investigated flow region, which enabled an increased recording rate of $10 \mathrm{~Hz}$. The camera was equipped with a Nikon $60 \mathrm{~mm}$ focal objective with $f_{\#}=8$, in combination with a narrow-bandpass $532 \mathrm{~nm}$ filter in order to minimize ambient light interference. The flow was imaged in the streamwise $(x)$ and wall-normal $(y)$ directions over a field of view of $129 \times 40 \mathrm{~mm}^{2}(6.5 \delta \times 2.0 \delta)$, resulting in a digital resolution of approximately 11 pixels $\mathrm{mm}^{-1}$. A dataset of order 1000 velocity vector fields was acquired. The recorded images were interrogated using a cross-correlation algorithm with iterative multi-grid window deformation (see Scarano \& Riethmuller 2000), using rectangular windows of final size $21 \times 17$ pixels $\left(1.9 \times 1.6 \mathrm{~mm}^{2}\right)(0.1 \delta \times 0.08 \delta)$, with an overlap factor of $75 \%$. This results in a vector spacing of about $0.5 \mathrm{~mm}(0.025 \delta)$. Postprocessing involved identifying erroneous vectors using the universal median test (see Westerweel \& Scarano 2005) with a maximum median deviation set at 2.5. The number of spurious vectors was less than $3 \%$ in the dataset. Table 2 summarizes the PIV recording parameters.

\section{Proper orthogonal decomposition}

In the present study, the fluctuating velocity field with respect to the estimated mean pattern is decomposed into a limited number of modes using the POD. The interested reader is referred to the review by Berkooz et al. (1993) for a more complete mathematical outline of the POD and its application to turbulent flows. Briefly, $N$ data measurements are simultaneously taken at $M$ time instants $t_{n}$, such that the samples are uncorrelated in time. The data represent instantaneous velocity fields obtained from PIV. The mean velocity at a point $\bar{u}(\boldsymbol{x})$ is subtracted from the instantaneous velocity $u\left(\boldsymbol{x}, t_{n}\right)$, leaving only data containing fluctuations from the mean $u^{\prime}\left(\boldsymbol{x}, t_{n}\right)$. The POD extracts $k$ time-independent orthonormal basis functions, empirical eigenfunctions or eigenmodes $\psi_{k}(\boldsymbol{x})$ and time-dependent orthonormal amplitude coefficients $a_{k}\left(t_{n}\right)$ such that the reconstruction

$$
u^{\prime}\left(\boldsymbol{x}, t_{n}\right)=\sum_{k=1}^{M} a_{k}\left(t_{n}\right) \psi_{k}(\boldsymbol{x})
$$

is optimal, in the sense that the functions $\psi$ maximize the normalized averaged projection of $\psi$ on to $u^{\prime}$, viz.

$$
\max _{\psi} \frac{\left\langle\left|\left(u^{\prime}, \psi\right)\right|^{2}\right\rangle}{\|\psi\|^{2}} .
$$

Here, $\|\cdot\|$ denotes the $L^{2}$-norm $\|f\|^{2}=(\cdot, \cdot)$, where $(\cdot, \cdot)$ is the standard Euclidean inner product $;|\cdot|$ is the modulus. The ergodic hypothesis is invoked so that ensemble 
averages $\langle\cdot\rangle$ are considered as time averages. The problem can be recast as the solution to the following Euler-Lagrange integral equation:

$$
\int_{\Omega} \boldsymbol{R}\left(\boldsymbol{x} ; \boldsymbol{x}^{\prime}\right) \psi\left(\boldsymbol{x}^{\prime}\right) \mathrm{d} \boldsymbol{x}^{\prime}=\lambda \psi(\boldsymbol{x}),
$$

where $\Omega$ is the flow domain; $\lambda$ is the eigenvalue; and the kernel $\boldsymbol{R}\left(\boldsymbol{x} ; \boldsymbol{x}^{\prime}\right)$ is the averaged space-correlation tensor, which for $M$ realizations of data at a finite number of discrete points is given by

$$
\boldsymbol{R}\left(\boldsymbol{x} ; \boldsymbol{x}^{\prime}\right)=\frac{1}{M} \sum_{n=1}^{M} u^{\prime}\left(\boldsymbol{x}, t_{n}\right) u^{\prime}\left(\boldsymbol{x}^{\prime}, t_{n}\right) .
$$

The non-negative and self-adjoint properties of $\boldsymbol{R}\left(\boldsymbol{x} ; \boldsymbol{x}^{\prime}\right)$ ensure that all eigenvalues are real and non-negative and can therefore be ordered, such that $\lambda_{n} \geqslant \lambda_{n+1} \geqslant \ldots \geqslant 0$. In the present study, we use the method of snapshots as first proposed by Sirovich (1987), to make use of the fact that $u^{\prime}\left(\boldsymbol{x}, t_{n}\right)$ and $\psi_{k}(\boldsymbol{x})$ span the same linear space. This enables the POD eigenmodes to be written as a linear sum of the data vectors,

$$
\psi_{k}(\boldsymbol{x})=\sum_{n=1}^{M} \Phi_{n}^{k} u^{\prime}\left(\boldsymbol{x}, t_{n}\right), \quad k=1, \ldots, M,
$$

where $\Phi_{n}^{k}$ is the $n$th component of the $k$ th eigenvector. The eigenmodes can then be found by solving the following eigenvalue problem:

$$
\mathbf{C} \Phi=\lambda \Phi .
$$

In this way, the eigenvectors of the $N \times N$ matrix $\boldsymbol{R}$ are found by computing the $M \times M$ correlation matrix $\mathbf{C}$ - an attractive method in the case in which $M \ll N$, as applies in the present study. (That is for the POD analysis $M \sim 500$, whereas each snapshot contains vector fields of size $N=271 N x \times 116 N y \sim 31000$. These values were determined primarily by computer memory limitations.) The reconstruction of a snapshot using a truncated subset of $K$ modes can be performed with

$$
u\left(\boldsymbol{x}, t_{n}\right)=\bar{u}(\boldsymbol{x})+\sum_{k=1}^{K} a_{k}\left(t_{n}\right) \psi_{k}(\boldsymbol{x}) .
$$

The total energy is defined as the mean square fluctuating value of velocity and is given by the sum of the eigenvalues $\lambda_{k}$. Each eigenmode may then be assigned an energy percentage $E_{k}$ based upon the eigenmode's eigenvalue, such that

$$
E_{k}=\lambda_{k} / \sum_{n=1}^{M} \lambda_{n}
$$

In compressible flows, the best choice of norm is not intuitively obvious: many of the (interrelated) variables, including thermodynamic quantities, may be important (see e.g. Rowley, Colonius \& Murray 2004 for a further discussion). In the present study, the use of the mean square fluctuating value of velocity, which represents the 'kinetic energy' of the flow, will be sufficient for our present purposes. 


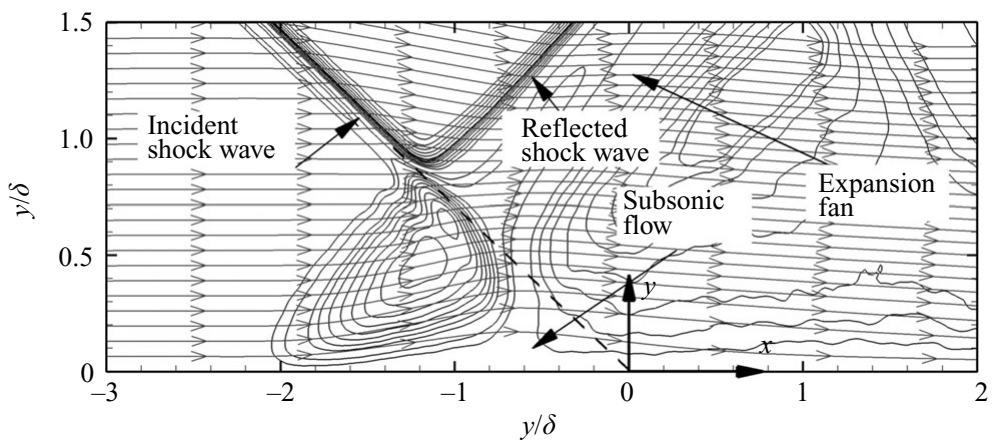

Figure 2. Overview of the mean flow organization of the interaction. Mean velocity streamlines are shown along with mean wall-normal velocity contours. Origin of the coordinate system is located at the extrapolated wall impingement point of the incident shock wave. Note the non-uniform axes. Adapted from Humble et al. (2007).

\section{Results and discussion}

\subsection{Mean flow organization of the interaction}

In order to provide the context for the main results later on, an overview of the interaction's mean flow organization is first presented in figure 2, which shows two-dimensional velocity streamlines complemented with mean wall-normal velocity contours, adapted from Humble et al. (2007). The spatial coordinates are normalized with the undisturbed boundary layer thickness $\delta$. Note also the non-uniform axes. The dashed line is the extrapolation of the incident shock wave to the wall and is taken to be the origin of the coordinate system used in the present study, with $x$ measured in the downstream flow direction and $y$ normal to the wall.

The streamlines verify uniform upstream flow and illustrate the overall distortion of the flow field, as a result of the interaction process. Regions of flow compression typically appear as relatively densely spaced wall-normal velocity contours, whereas regions of flow expansion typically appear as relatively sparsely spaced wall-normal velocity contours. Notice how, close to the wall, the distance between adjacent streamlines increases throughout the interaction, whereas farther away from the wall, the distance between adjacent streamlines decreases. This highlights the contrasting behaviour between the subsonic and supersonic parts of the flow field, respectively, and gives us our first hints of a multi-structured interaction. Observe also the compression waves generated within the incoming boundary layer approximately $2 \delta$ upstream of the origin. These compression waves coalesce as they leave the boundary layer to form the reflected shock wave, which will become a focal point of our discussion later on.

Farther downstream, the subsonic layer begins to contract, causing the outer fluid to move back towards the wall. Although difficult to discern from this figure, a gradual recompression process takes place in the outer flow as it is slowly turned back parallel with the wall. Importantly, for what follows, the time-averaged velocity field (see Humble et al. 2007) strongly suggests that no flow separation is observed on the mean. Given the difficulties of resolving very close to the wall with PIV, however, this is by no means definitive, although this does not affect the overall conclusions to be drawn. 

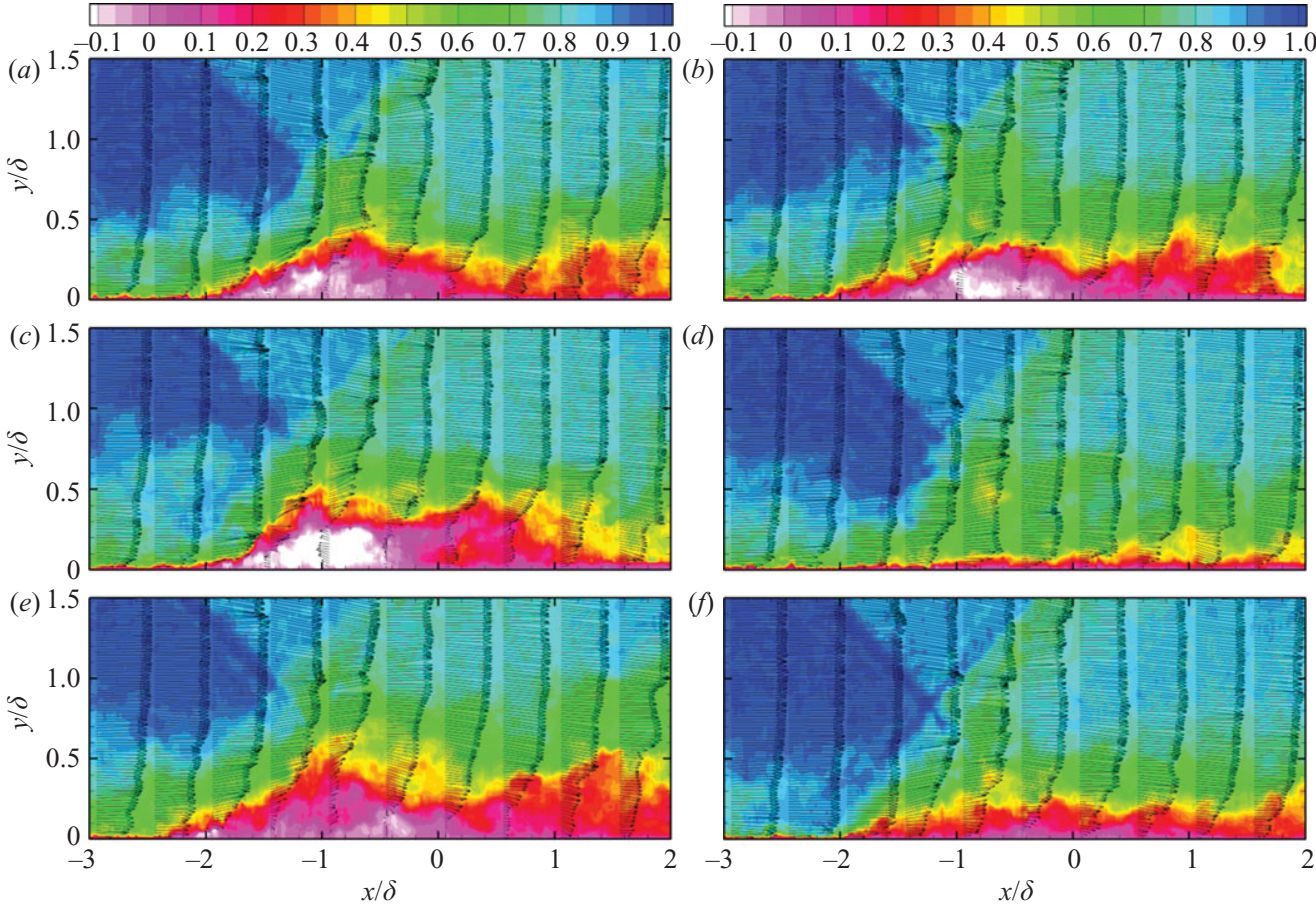

FIGURE 3. Instantaneous flow organization of the interaction. A series of uncorrelated instantaneous streamwise velocity distributions $u / U_{\infty}$ are presented. Velocity vectors show 1 in 22 in the streamwise direction. Note the non-uniform axes.

\subsection{Instantaneous flow organization of the interaction}

The question now naturally arises as to whether the above result is an accurate representation of the flow structure, since it is the result of time averaging the flow fields. A series of instantaneous streamwise velocity fields is therefore shown in figure 3. These fields are chosen because they exemplify the features observed in the complete dataset. The acquisition time between any two velocity fields $(10 \mathrm{~Hz}$ recording rate) is much greater than any characteristic time scale, and so the velocity fields are uncorrelated in time. Velocity vectors are also shown, under-sampled with 1 in 22 in the streamwise direction for clarity. Note also the non-uniform axes.

The results portray an unsteady incoming boundary layer, with its instantaneous edge appearing as an intermittent interface with the outer free-stream flow. This result is similar to those of Smith \& Smits (1995), who visualized the structure of supersonic boundary layers using Schlieren and Rayleigh scattering and characterized the outer layer as consisting of an array of regularly spaced uniform low-density bulges, separated from a uniform higher-density free-stream by a sharp, instantaneously ragged interface. Moving downstream, the penetration of the incident shock wave into the boundary layer can be observed. Note the relatively weak feature immediately upstream of the incident shock wave (roughly parallel to it) in figure $3(e, f)$, for example. This is due to optical aberration effects introduced by the inhomogeneous index of refraction of this compressible flow.

The global structure of the interaction can be seen to change significantly in time. Figure $3(a-c)$ shows that in contrast to the mean velocity distribution, fluid close to the wall is instantaneously redirected upstream as it approaches the interaction, leading 
to the formation of a separated flow region. At these instants, the flow structure is very different from the mean flow field and resembles the mean separated flow model, as characterized by a well-defined separation bubble (see Simpson 1989). The size of the separation bubble clearly varies in time. By visual inspection, it appears to have a streamwise length that varies in the range 0-2 (compare for instance figure $3 c, d)$. The flow within the bubble cannot be characterized as a stagnant velocity zone; the maximum backflow velocity often exceeds $0.1 U_{\infty}$. This is consistent with the measurements made by Bookey, Wyckham \& Smits (2005) in their incident SWTBLI at Mach 2.9; they reported a similar reversed-flow velocity magnitude. In contrast, figure $3(d, f)$ shows instants at which the boundary layer remains fully attached throughout the interaction. The interaction may therefore be conceived as an intermittently separated configuration that is very different from its time-averaged counterpart.

On closer inspection, figure $3(a-c, e)$ shows that the interaction exhibits, on an instantaneous basis, a multi-layered structure, characterized by a high-velocity outer layer (typically $u / U_{\infty}>0.5$, shown in green-blue), above a layer of relatively low velocity close to the wall (typically $u / U_{\infty}<0.5$, shown in red-orange). These layers are conceptually reminiscent of the zones of relatively uniform momentum reported by Adrian, Meinhart \& Tomkins (2000) in their incompressible turbulent boundary layer. The term 'layer' is used here to emphasize that while they are defined instantaneously, they typically extend in the streamwise direction across the measurement domain. The outer layer comprises most of the incoming boundary layer. The inner layer thickens rapidly as it enters the first part of the interaction, often reaching its maximum thickness beneath the incident shock wave. The reader should note, however, that the above is somewhat of a subjective characterization, given the complex nature of the interaction, and is done so for the purposes of simplifying the conceptual interpretation of the results.

Nevertheless, the two layers appear to be separated by a thin region of relatively high shear, indicated by the yellow contour. Figure $3(b, c, e)$ shows that this interface has an irregular and intermittent nature, with a typically downstream-sloping pattern within the redeveloping boundary layer. Figure $3(b, c)$ shows that a vigorous mixing occurs across these layers, with outer fluid injected deep into the low-velocity region. The flow in the detached shear layer therefore appears to share superficial similarities with the plane mixing layer, as noted by $\mathrm{Na} \&$ Moin (1998) in their DNS of an incompressible separated flow, for example. Moving downstream, figure 3(a-c) shows that the separated shear layer undergoes a local instantaneous reattachment. Yet while the reattachment process takes place within a relatively short streamwise extent, the overall velocity deficit within the inner layer persists much farther downstream. In fact, it is clear that the boundary layer does not fully recover to its incoming conditions within the present measurement domain.

\subsection{Vorticity characteristics}

To complement the discussion on the instantaneous velocity fields, out-of-plane vorticity and vortical (or coherent) structures within the interaction are visualized. This constitutes one of the subtler aspects of interpreting the PIV velocity vector fields (see Adrian et al. 2000). The instantaneous velocity fields in figure 3 are reproduced in figure 4, now displaying flooded contours of instantaneous out-of-plane (spanwise) vorticity: $\omega_{z} \delta / U_{\infty}$. Note the non-uniform axes. To ease visualization, the spatial derivatives have been estimated by a second-order least squares regression using a $5 \times 5$ kernel, although this does not affect the conclusions to be drawn. Overlaid 


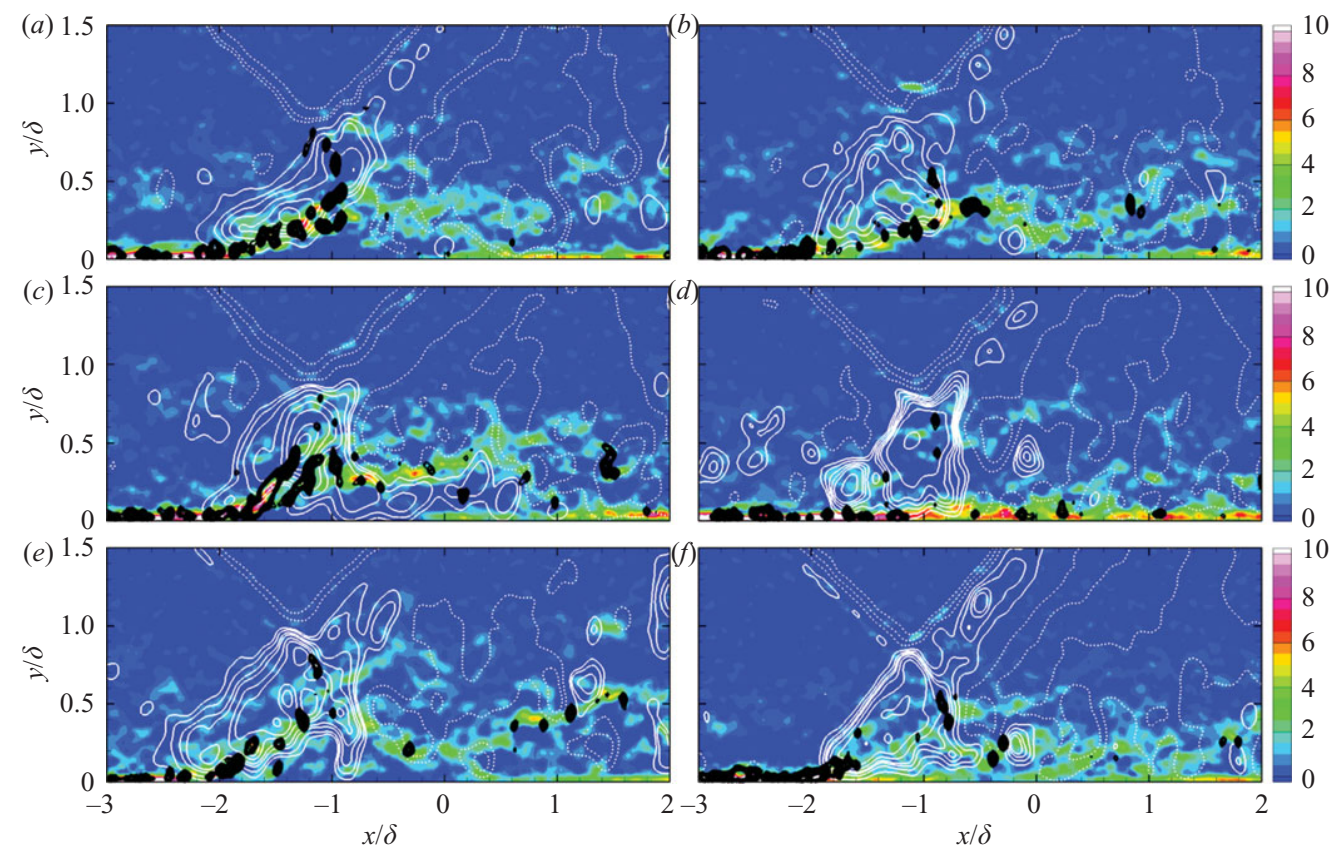

FIGURE 4. Instantaneous vorticity structure of the interaction. Discrete regions of $Q>0$ (determined using the $Q$-criterion) are shown in black along with flooded out-of-plane vorticity contours $-\omega_{z} \delta / U_{\infty}$. Wall-normal velocity contours are shown as white lines with 12 levels within the range $-0.15 \leqslant v / U_{\infty} \leqslant 0.15$. The dashed contours indicate negative values. All figure parts correspond to those in figure 3 .

are white lines of instantaneous wall-normal velocity $\left(v / U_{\infty}\right)$ within the range -0.15 $\leqslant v / U_{\infty} \leqslant 0.15$, where dashed lines indicate negative values.

In addition, following the method proposed by Hunt, Wray \& Moin (1988), vortical structures are identified, as being any contiguous region of flow in which the second invariant of the velocity gradient tensor $Q$ is greater than zero. In the present study, it is assumed that the criterion $Q>0$ is valid in compressible flow, since the (in-plane) divergence of the velocity field was found to be relatively small (typically an order of magnitude less than the rate of strain and rate of rotation), except of course in the presence of shock waves, but more importantly, because results were obtained that were very similar to the use of other (incompressible) criteria, such as the $\lambda_{2}$ criterion (see Jeong et al. 1997). The reader will also note that in two-dimensional measurements there are missing components of the velocity gradient tensor, and the following determined patterns must therefore be considered as only a section of the complete three-dimensional structure from the $x-y$ plane. Furthermore, since the present resolution cannot be used to accurately determine statistics of derived quantities such as vorticity, the following results are used qualitatively to illustrate the interaction's global vorticity organization.

With these caveats in mind, figure 4 shows some interesting features. Within the incoming boundary layer, most of the vorticity tends to be concentrated relatively close to the wall in the form of a vorticity layer. This is consistent with the work of Ringuette, Wu \& Martin (2008), who visualized instantaneous out-of-plane vorticity from the DNS of their supersonic boundary layers in the streamwise-wall-normal 
plane and also observed regions of relatively high levels of vorticity close to the wall. It was also shown by these authors when visualizing the same result in three dimensions that the attendant vortical structures are in fact connected out of the plane and form complicated hairpin packets. Our two-dimensional patterns must therefore be considered as only a cross-sectional view of a more complex three-dimensional flow field. In the present results, flow structures revealed by the $Q$-discriminant are also distinguished within the incoming boundary layer very close to the wall, although the reader must exercise caution when interpreting such structures. The present resolution is insufficient to properly resolve the swirling motion in this region, and many are most likely to be numerical artefacts.

As the interaction is approached, the flow field structure can be seen to change dramatically. Figure $4(a-c)$ shows that when flow separation occurs, the vorticity layer and vortical structures are lifted up away from the wall into the shear layer within the detachment region. They appear to turn around the separation bubble, as revealed by a comparison of figures $3(b, c)$ and $4(b, c)$ for instance. This is in agreement with the observations made by $\mathrm{Na} \&$ Moin (1998) and Chong et al. (1998) in their DNS of incompressible separated flows, who observed that such structures would treat the separation bubble essentially like a streamlined obstacle and would often impinge back on to the wall farther downstream. The present vortical structures are lifted away from the wall and appear to propagate mainly through the incident shock wave's tip. This is consistent with the DNS of an incident SWTBLI by Pirozzoli \& Grasso (2006), who found that the oscillatory motion of the incident shock wave occurs mainly at its tip. Farther downstream, the vortical structures return to the proximity of the wall in the reattachment region. Notably, very few vortical structures can be observed within the separated flow region itself and instead typically occur along the interface between the two layers.

It is also interesting to observe that a similar lifting of the vorticity layer and vortical structures occurs even without a significantly reversed-flow region (see e.g. figure $4 e$ ). This was observed many times throughout the dataset. It therefore appears that the vortical structures respond to a strongly retarded inner layer flow, in much the same way as they do to a large-scale recirculating bubble. Accordingly, when there is no significant inner layer velocity deficit, there is no observed lifting of the vortical structures, as demonstrated in figure $4(d)$, for instance. This is especially important in the present interaction because it means that it can instantaneously exhibit many of the features of a large-scale separated interaction, even without a large-scale reversed-flow region.

Farther downstream, regions of spanwise vorticity can be seen to spread more broadly over the height of the interaction with downstream development. Figure $4(a, b, e)$ shows that they often appear as interconnected chains, in combination with relatively isolated regions that are dispersed throughout the height of the boundary layer. Spatio-temporal studies of an incident SWTBLI by Dupont et al. (2006) have determined that frequencies tend to decrease along the separated flow region, noting that in subsonic recirculating flows, such frequency evolutions are associated with large-scale structures that are convected in a mixing layer that develops downstream of flow detachment. It therefore appears possible that vortical structures grow and develop within the separated shear layer, as part of a vortexshedding process associated with the separation bubble. This will become a focal point of the POD analysis later on. 
The wall-normal velocity contours in the first part of the interaction are especially interesting because they indicate a zone of compression that is the reflected shock wave region. This zone can be observed to change significantly in time. It can appear as a relatively diffuse feature, such as in figure $4(e)$, whereas at other instants it appears relatively distinct, such as in figure $4(f)$. In addition, lines of wall-normal velocity often appear instantaneously warped. Figure 4(a) shows a concave compression region, whereas figure $4(b)$ shows a convex compression region. At other instants, the reflected shock wave region assumes a combination of both these patterns, as shown in figure $4(c)$, for example.

To gain an idea of the time scales of this unsteadiness, hot-wire anemometry measurements were carried out within the centre of the reflected shock wave region within the boundary layer at $x / \delta=-1.8$ and $y / \delta=0.2(y=4 \mathrm{~mm})$. This location was chosen because the flow here is supersonic, which avoids the complexities of transonic flow, but is sufficiently close to the wall to make comparisons with results from the literature. The results will be presented in more detail in a later paper, but it was found that peak energetic frequencies occur at about $1-3 \mathrm{kHz}\left(0.04-0.12 U_{\infty} / \delta\right)$, substantiating the notion that frequencies within the reflected shock wave region are at least an order of magnitude lower than the characteristic boundary layer frequency $U_{\infty} / \delta$ (see e.g. Dussauge et al. 2006).

Now consider the Strouhal number $S_{L}=f L / U_{\infty}$, where $L$ is defined as the distance between the extrapolated wall impingement points of the reflected and incident shock waves (see Dupont et al. 2006); then in the present study $L / \delta=2.27(L=$ $45 \mathrm{~mm}$ ), which results in an energetic Strouhal number range $S_{L}=0.1-0.27$, about 3-10 times larger than the most energetic Strouhal numbers reported elsewhere. In particular, the compilation of $S_{L}$ by Dussauge et al. (2006) for several configurations in the literature in which experimental data were available, including compression ramp and blunt fin interactions as well as over-expanded nozzles, revealed that $S_{L}$ grouped the experiments together for about $S_{L}=0.02-0.05$. This discrepancy is currently an active area of research, but the intermittent nature of the interaction may provide a possible explanation: introducing $L_{r m s}$ as the r.m.s. of the (largest) instantaneous separation bubble length, which includes the cases in which no bubble occurs, gives $L_{r m s} / \delta=0.28$, which results in $S_{L}=0.01-0.03$, in much better agreement with the results reported above. In the absence of further results, however, the data remain provocative. Nevertheless, the fact remains that the reflected shock wave region is dominated by energetic frequencies that are an order of magnitude lower than those within the undisturbed boundary layer at the same distance from the wall, consistent with what has been reported in the literature.

Returning now to investigate in more detail the role of the vortical structures in the separation process, figure $4(b)$ is repeated in figure $5(a)$, this time with velocity vectors in a convective frame of reference of $0.8 U_{\infty}$. In this frame of reference, many of the velocity vectors can be seen to swirl around distinct regions of $Q$ within the detachment region. It appears from these convective velocity vectors that a fluid exchange occurs between the inner and outer layers of the interaction. To better illustrate this, the instantaneous wall-normal velocity is extracted along the thick grey line $S$, which is judiciously chosen to be the locus of points through the vortical structures as they propagate throughout the interaction and is shown in figure $5(b)$. The instantaneous separation and reattachment points $\left(x_{s}\right.$ and $x_{r}$ respectively; see $\S 4.4$ for further details), as well as the distribution of the mean wall-normal velocity extracted along $S$, are also shown for comparison. 


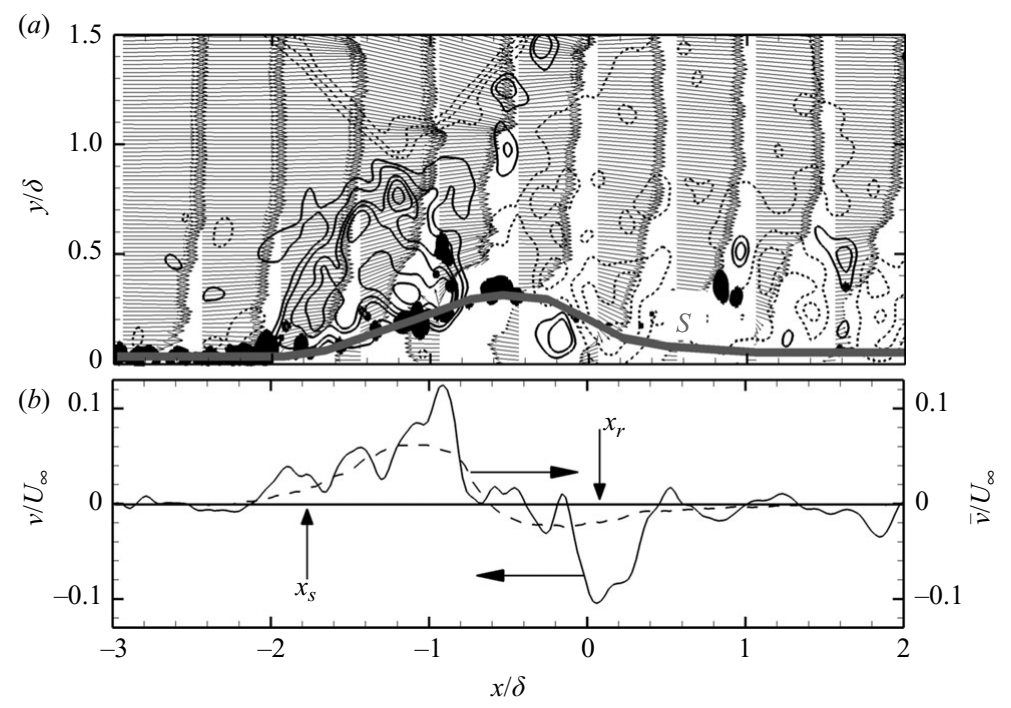

FIGURE 5. Instantaneous vortical structure distribution of the interaction. (a) Instantaneous velocity field taken from figure 4(b) (see the caption for figure 4) with velocity vectors shown in a convective frame of reference of $0.8 U_{\infty},(b)$ Mean (dashed line) and instantaneous (solid line) wall-normal velocity profiles extracted along $S$. Arrows assign data to the appropriate axes.

It can be seen that both the instantaneous and mean wall-normal velocities remain approximately zero within the incoming boundary layer. Within the first part of the interaction, the instantaneous velocity profile begins to deviate from the mean profile, characterized by a sinuous alternation of positive and negative wall-normal velocity fluctuations. Maximum instantaneous wall-normal velocities reach over $10 \%$ of $U_{\infty}$, which is in fact comparable to the maximum (streamwise) reversed-flow velocity. A closer comparison of figure $5(a)$ with figures $3(b)$ and $4(b)$ suggests a physical mechanism, whereby high-velocity fluid is drawn in and ingested between the vortical structures as they ride over the inner flow, whereas low-velocity fluid from the separated flow is transported away from the wall. This description is not dissimilar to the scenario described by Simpson (1989) in low-speed incompressible separated flows, who discussed how movies of laser-illuminated smoke and turbulence energy results clearly reveal how large-scale eddy structures supply most of the near-wall reversed flow. The separation process of the present interaction therefore appears to share some important similarities with incompressible separated flows, and we will discuss later on how these structures may be reconciled with the large-scale unsteadiness.

\subsection{Statistical analysis}

It should now be clear from the preceding results that the structure of the interaction changes significantly in time, and to make more quantitative statements we characterize this behaviour statistically. To begin with, figure 6 shows lines of constant value for the reversed-flow probability of the instantaneous streamwise velocity being less than zero $p(u<0)$. These contours have been computed from histograms of the instantaneous streamwise velocity. For the sake of clarity, the inset shows a zoomed view of the interaction region, with a dilated wall-normal axis. The region of $p(u<0)$ appears as a relatively shallow region that is elongated in the streamwise direction and roughly symmetric about an axis in the wall-normal direction. This 


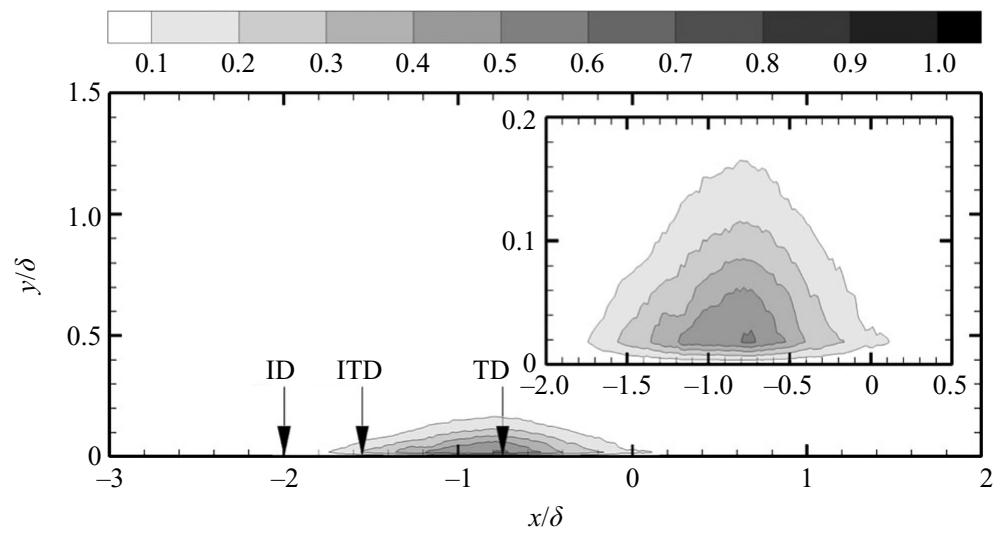

FIGURE 6. Regions of equiprobability of reversed flow within the interaction $p(u<0)$. The terms ID, ITD and TD are defined in the text. The inset shows zoomed region with the wallnormal coordinate dilated for clarity.

confirms an intermittently separated flow region with a streamwise extent within $0-2 \delta$. Observe how $p(u<0)$ is never equal to unity anywhere in the flow, consistent with the observation that no reversed flow occurs on average. On the other hand, $p_{\max }(u<0)$ within the interaction is about 0.5 , indicating that flow reversal occurs in around $50 \%$ of the total number of realizations. Of course, this inference is a conservative estimate, since reversed flow may occur in different regions. Note that because of the limited number of contour levels displayed, no reversed flow is shown within the incoming and redeveloping boundary layers.

The existence of an intermittent reversed flow complicates the criterion used to define separation, and we need to distinguish between mean and instantaneous flow separation. For steady free-stream separating boundary layers, the following set of quantitative definitions on the separation state near the wall have therefore been proposed by Simpson (1989): 'incipient detachment' (ID) occurs when the instantaneous backflow occurs $1 \%$ of the time; 'intermittent transitory detachment' (ITD) occurs with instantaneous backflow $20 \%$ of the time; 'transitory detachment' (TD) occurs with instantaneous backflow $50 \%$ of the time; and 'detachment' (D) occurs where the time-averaged wall-shear stress $\bar{\tau}_{\text {wall }}=0$. In the present study, the locations of ID, ITD and TD occur at approximately $(x / \delta)_{I D}=-2.0,(x / \delta)_{I T D}=-1.6$, and $(x / \delta)_{T D}=-0.75$, respectively, and are indicated in the figure, where ensemble averages have been interpreted as time averages. The location $\mathrm{D}$ does not exist in the present interaction, but its occurrence for a sufficiently stronger interaction is likely to be very close to TD (see Simpson 1989). Overall, it is clear that depending on the criterion used, there is quite some discrepancy in the location of the separation point.

As a result of the interaction's intermittency, the instantaneous separation and reattachment points wander back and forth along the wall in time. To characterize this behaviour, a statistical analysis of the instantaneous streamwise location of the separation point $x_{s}$ and reattachment point $x_{r}$ is carried out. Following the approach of Kiya \& Sasaki (1985), the reversed-flow region is defined as a contiguous region based on where the instantaneous streamwise velocity near the surface becomes negative, and we determine $x_{s}$ and $x_{r}$ as the zero crossings of the instantaneous streamwise velocity. The velocity distribution at the height $y=1.0 \mathrm{~mm}(y / \delta=0.05)$ was selected, although the overall trends of the results to be discussed were found to be relatively 

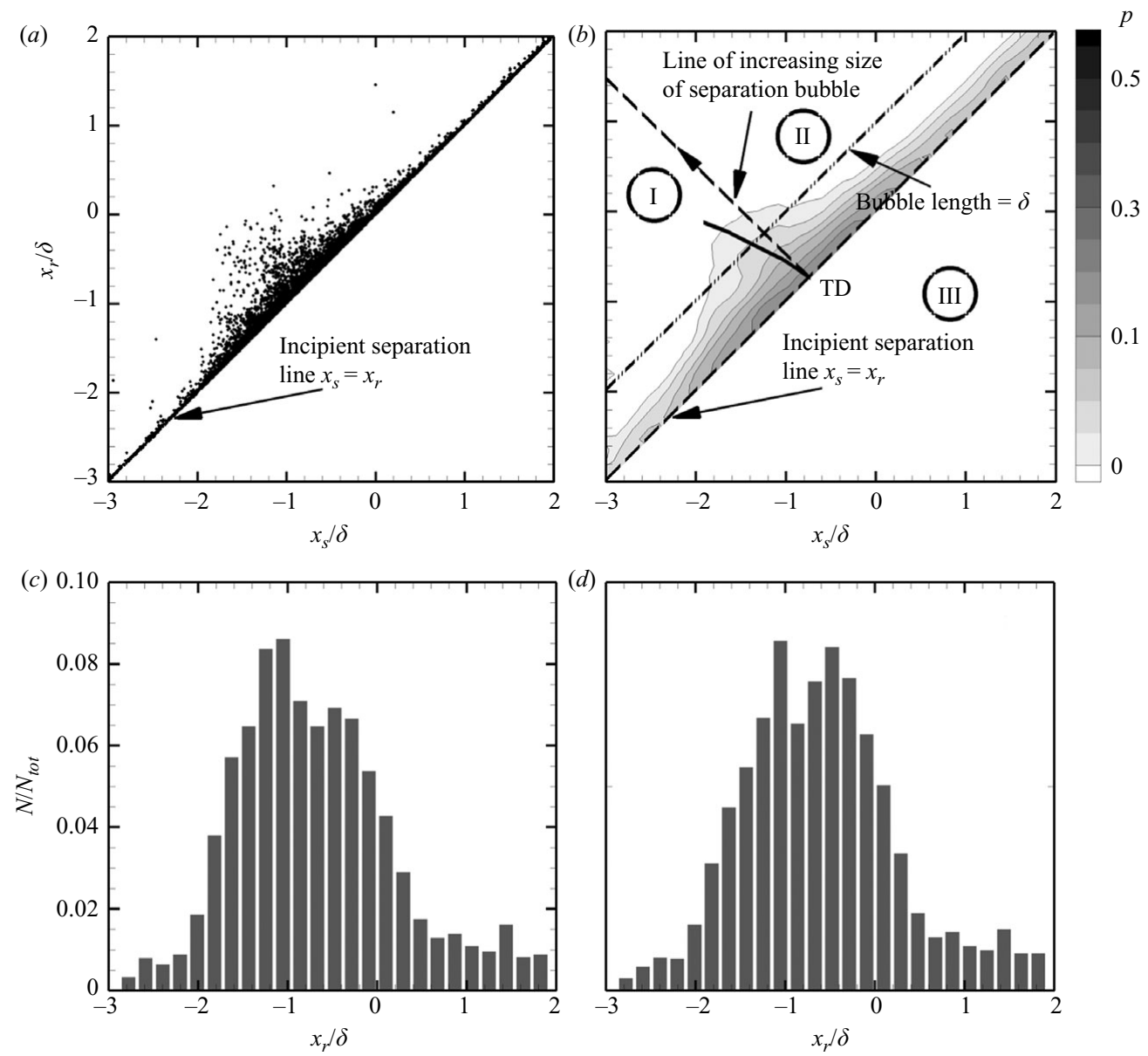

FigurE 7. Statistical analysis of the instantaneous separation point $x_{s} / \delta$ and reattachment point $x_{r} / \delta,(a) x_{s} / \delta$ compared with $x_{r} / \delta,(b)$ joint p.d.f.s of $x_{s} / \delta$ and $x_{r} / \delta$ showing the behaviour of the separated flow region, $(c)$ p.d.f. of $x_{s} / \delta,(d)$ p.d.f. of $x_{r} / \delta$.

insensitive to small changes in the height chosen. Figure 7(a) shows $x_{s}$ compared with $x_{r}$. The line $x_{s}=x_{r}$ delimits the case in which the separation and reattachment points coincide (i.e. in which incipient separation occurs). The accumulation of many points along this line suggests that many small-scale separation regions occur, and inspection of the instantaneous realizations reveals that these regions are often present at several locations simultaneously along the wall. Given the present resolution, however, some of these relatively small-scale separation regions should be considered as numerical artefacts.

Discrete joint probability density functions (p.d.f.s) are shown in figure $7(b)$, which indicate the relative probability of the $i$ th streamwise position $(x / \delta)_{i}$ of separation and reattachment falling within a range $(x / \delta)_{1}<(x / \delta)_{i}<(x / \delta)_{1}+\Delta(x / \delta)$, where $\Delta(x / \delta)$ is the bin width of the p.d.f., taken as approximately 0.2 in the present study. These contours have been computed from histograms of the instantaneous streamwise velocity, and therefore $p$ includes the cases in which the boundary layer remains fully attached. From these results, we may arbitrarily delineate the behaviour of the separated flow into the following regions as indicated in the figure: 
(i) Region I. In this region $x_{s}<x_{r}$, and the separated flow region is relatively upstream of the interaction. That is if the separation location is relatively upstream, then it is more likely to be accompanied by a relatively upstream reattachment location.

(ii) Region II. In this region $x_{s}<x_{r}$, and the separated flow region is relatively downstream of the interaction. That is if the separation location is relatively downstream, then it is more likely to be accompanied by a relatively downstream reattachment location.

(iii) Region III. In this region $x_{s}>x_{r}$, which is a physically impossible case by convention, and so data points do not fall in this region.

The line perpendicular to the incipient separation line separates regions I and II and marks the case of a separation bubble changing size symmetrically about a plane perpendicular to the wall. It emanates from TD at $x / \delta=-0.75$. Points along this line from TD represent separation zones of increasing streamwise extent. As expected, $p$ rapidly decreases along this line, and no bubble size greater than $2 \delta$ was found to occur. Lines of equiprobability appear quasi-symmetric about this line, indicating that for an increasing bubble size, it is more likely that $x_{s}$ appears relatively upstream and $x_{r}$ appears relatively downstream, and vice versa. This is consistent with the notion of an expanding and contracting separation bubble and with the covariance coefficient distribution of the separation and reattachment locations obtained by Pirozzoli \& Grasso (2006), which also suggests the occurrence of an expanding and contracting separation bubble. A trend line showing the locus of points of maximal probability is also shown in figure 7(b) for comparison, indicated by the solid line. This line can be seen to deviate slightly into region I from the straight line emanating from TD, indicating the intriguing trend that for an increasing bubble size, its centre appears slightly more upstream, in the time-averaged sense.

Discrete p.d.f.s of $x_{s}$ and $x_{r}$ in figure 7(c,d) show that both locations occur along the complete streamwise extent of the measurement domain. The ID location at $x / \delta=-2.0$ in particular seems to mark the onset of a significant fraction of separated flow cases and roughly corresponds to the region in which the boundary layer first encounters the upstream influence of the interaction. Observe how the p.d.f. tails persist appreciably farther downstream of the interaction than they do upstream, indicating that reversed-flow regions occur more frequently within the redeveloping boundary layer than within the incoming boundary layer. The r.m.s. values of $x_{s}$ and $x_{r}$ are approximately 0.3 and 0.29 , respectively, indicating that the separation and reattachment points undergo somewhat similar degrees of spatial variation. This may be compared with the results of Pirozzoli \& Grasso (2006), who found a larger variation of the reattachment location, and $\mathrm{Na} \&$ Moin (1998), who found a larger variation of the separation location.

\subsection{On the cause ( $s$ ) of the interaction's unsteadiness}

Having outlined some physical and statistical properties of the interaction's unsteadiness, we now wish to shed some light on the possible mechanism(s) behind this behaviour. As can probably be discerned from the introduction, however, the extant literature on SWTBLI unsteadiness is too large and diverse to be surveyed meaningfully within the confines of this paper. Nevertheless, we wish to carefully distil some pertinent works that will help guide us on attempting to consolidate the present results. In particular, Beresh et al. (2002) have examined the role of the incoming boundary in a compression ramp interaction by considering incoming boundary layer velocity profiles derived from PIV data, conditioned on the instantaneous separation 
(a)

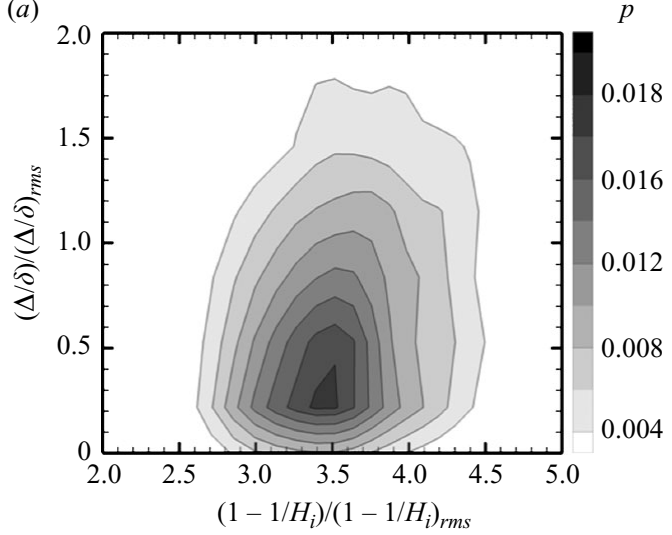

(b)

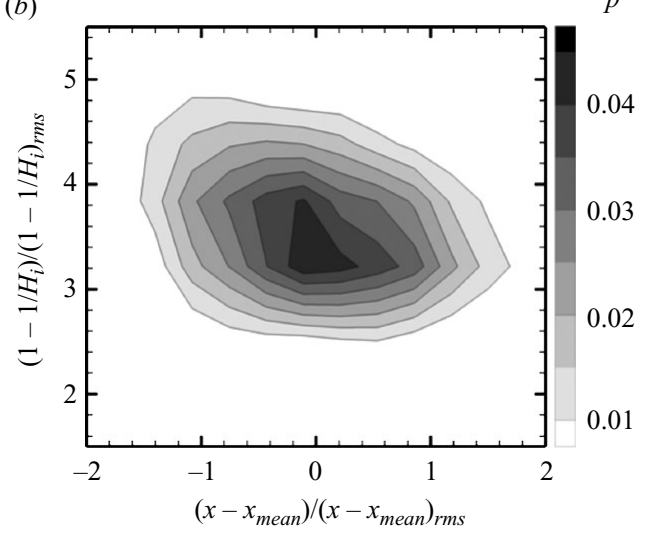

FIGURE 8. Relationship between the incoming boundary layer and interaction region unsteadiness. (a) Joint p.d.f. of equilibrium shape factor $1-1 / H_{i}$ and streamwise extent of separation bubble $\Delta / \delta$, (b) Joint p.d.f. of $1-1 / H_{i}$ and streamwise location of the reflected shock wave $\left(x-x_{\text {mean }}\right) / \delta$. Shape factor $H_{i}$ is computed at $x / \delta=-3.0$, and only the largest value of $\Delta$ in each realization is considered. Note that the results are normalized by their respective r.m.s. values.

shock foot location derived from wall-pressure measurements. Ensemble-averaged velocity fluctuations conditioned on different types of shock motion revealed that positive fluctuations in streamwise velocity near the wall corresponded to downstream shock motion, and vice versa. Since positive fluctuations within the boundary layer suggest that its profile is momentarily fuller, and vice versa, a suitable proposal was that the shape parameter, which is a direct measure of the fullness of the boundary layer's streamwise velocity profile, is first related to the size of the separated flow region.

To characterize the state of the incoming boundary layer in the present study, we use the 'equilibrium shape parameter', defined as $1-1 / H_{i}$, where $H_{i}$ is the incompressible shape parameter. The use of $1-1 / H_{i}$ is particularly well suited to SWTBLIs involving large-scale separated flows (see Délery \& Marvin 1986). The shape parameter $H_{i}$ was determined by integrating the incoming streamwise velocity profiles at the upstream edge of the measurement domain $x / \delta=-3.0$. The trends to be discussed, however, were found to be relatively insensitive to small variations in the streamwise location chosen, consistent with the long tails observed in streamwise velocity autocorrelation function (see e.g. Ganapathisubramani, Clemens \& Dolling 2006). The size of the separated flow region is characterized by its streamwise extent $\Delta=x_{r}-x_{s}$. Since several reversed-flow regions often occur within a realization, only the largest value of $\Delta$ in each realization is considered (if reversed flow occurs at all).

Joint p.d.f.s between $1-1 / H_{i}$ and $\Delta$ are shown in figure $8(a)$, where they have been normalized by their respective r.m.s. values. The reader should exercise some caution when interpreting values of the shape parameter, due to the necessity of having to accurately resolve the velocity close to the wall. Based on a linear error propagation analysis, taking into account the r.m.s. measurement uncertainties of both the instantaneous streamwise velocity and PIV resolution, the estimated r.m.s. measurement uncertainty of $H_{i}$ is of the order $5 \%$. Indeed, figure $8(a)$ indicates that the median shape factor is $1-1 / H_{i} \approx 0.285$, from which it can be deduced that $H_{i} \approx 1.4$, consistent with the value for a zero-pressure-gradient turbulent boundary 
layer. Note also that $\Delta$ can only be positive or null: therefore its median value is positive.

It can be seen that the contours of equiprobability appear elliptical, with the major axis of the ellipse inclined with the abscissa. The nature of the contour levels, as presented here, can indicate that for a given $\left(1-1 / H_{i}\right) /\left(1-1 / H_{i}\right)_{r m s}$, a similar probability is obtained for large bubble sizes as well as for small ones. However, for bubble sizes larger than the median value, for such inclined contours and given the value of $\left(1-1 / H_{i}\right) /\left(1-1 / H_{i}\right)_{r m s}$, the probability of observing a larger bubble always decreases, independent of whether or not the value of $\left(1-1 / H_{i}\right) /\left(1-1 / H_{i}\right)_{r m s}$ is greater or less than its median value. (Strictly speaking, only for vertical contours would this not be the case.) This behaviour of course becomes more difficult, and more dubious, to discern the further one moves away from the median $\left(1-1 / H_{i}\right) /\left(1-1 / H_{i}\right)_{r m s}$, particularly for large values of $\left(1-1 / H_{i}\right) /\left(1-1 / H_{i}\right)_{r m s}$.

With these caveats in mind, although the elliptical appearance of the results depends somewhat on the scale chosen, the trend is then clear: when $1-1 / H_{i}$ is greater than its median value (boundary layer less full), the streamwise extent of the (largest) separated flow region is more likely to be larger than its median size. Conversely, when $1-1 / H_{i}$ is less than its median value (boundary layer fuller), the streamwise extent of the (largest) separated flow region is more likely to be smaller than its median size. Put another way, the larger the given bubble size, the smaller the probability $p$ of observing it. However, this probability decreases less rapidly along a line of slightly increasing $\left(1-1 / H_{i}\right) /\left(1-1 / H_{i}\right)_{r m s}$ extending from its median value than it does for the median value alone. The converse is thus that a slightly increasing $\left(1-1 / H_{i}\right) /\left(1-1 / H_{i}\right)_{r m s}$ leads to a higher probability of an increased bubble size than if only the median value is considered. It is important to emphasize, however, that the correlation coefficient between $1-1 / H_{i}$ and $\Delta$ is -0.22 , and so the reader must exercise some caution here and consider the above relationship to be weak although not negligible.

Nevertheless, these results are qualitatively consistent with the model proposed by Beresh et al. (2002), in the sense that a fuller incoming velocity profile entering the interaction provides an increased resistance to flow separation, which leads to a smaller separated flow region, and vice versa. It must be stressed, however, that given the limited correlation of the present data, this relationship may not be the most important mechanism present, and furthermore, it represents a quasi-steady view, in the sense that the incoming boundary layer profile and separated flow region are related, but no temporal delay is taken into account. On the other hand, as mentioned in the introduction, the time scales associated with the low-frequency unsteadiness are lower than the convective time by at least an order of magnitude, and so the quasisteady view is not therefore considered restrictive, at least not for the low-frequency unsteadiness of the interaction.

We can also anticipate that $1-1 / H_{i}$ is related to the reflected shock wave position, since the latter has been shown to undergo a displacement due to the expansion and contraction of the separation bubble (see e.g. Dolling \& Murphy 1983; Erengil \& Dolling 1993). After experimenting with a variety of criteria, it was found that the reflected shock wave position could be conveniently estimated from the instantaneous wall-normal velocity distribution across the shock wave by using the streamwise location at which $v / U_{\infty}=0$ as a proxy for the shock wave's position. This was because the trends to be discussed were found to be relatively insensitive to several other criteria, including more exotic quantities, such as the (in-plane) divergence. 
Figure $8(b)$ shows joint p.d.f.s of $1-1 / H_{i}$ and the instantaneous position of the reflected shock wave relative to its mean position $\left(x-x_{\text {mean }}\right) / \delta$. Note again that these data have been normalized by their respective r.m.s. values. The reflected shock wave undergoes a motion that has a streamwise extent of the order $0.5 \delta$ (shown as approximately four standard deviations in the figure), consistent with what is observed in the instantaneous realizations. The contours of equiprobability again appear elliptical, with the major axis of the ellipse inclined with the abscissa. This suggests that when $1-1 / H_{i}$ is less than its median value (boundary layer fuller), the reflected shock wave is more likely to be located downstream of its mean position. Conversely, when $1-1 / H_{i}$ is greater than its median value (boundary layer less full), the reflected shock wave is more likely to be located upstream of its mean position. The correlation coefficient between $1-1 / H_{i}$ and $\left(x-x_{\text {mean }}\right) / \delta$ is -0.3 , indicating again that this relationship is relatively weak although not negligible.

In a related work, Dupont et al. (2008) have recently carried out PIV measurements of their shock reflection interaction and found a correlation between the instantaneous position of the shock wave and thickness of the recirculating zone. They proposed that when the recirculating zone dilates, the shock wave moves upstream, and when the bubble contracts, the shock wave moves downstream. The correlation coefficient in their study was found to be -0.5 based on 200 realizations. In the present study, the calculation of the corresponding correlation coefficient is more complicated, both because the interaction is only intermittently separated, meaning that a separation bubble is not always present, and because when separation does occur, it is not always large scale (i.e. $O(\delta)$ ) but often includes several smaller-scale separated flow regions scattered simultaneously throughout the interaction.

The effects of computing the correlation coefficient conditioned on various bubble sizes are therefore especially interesting. Considering the complete dataset for instance, the corresponding correlation coefficient between the separation bubble size and shock wave location is about -0.1 . However, considering bubble sizes $\Delta / \delta>0.2$, which helps remove the influence of small-scale separation (as well as numerical artefacts) on the results, the corresponding correlation coefficient increases to between -0.2 and -0.3 depending on the $\Delta / \delta$ threshold used. (For higher thresholds of $\Delta / \delta>1.0$ (say), the limited number of data points becomes an important factor.) The idea of a breathing separation bubble displacing the shock wave is therefore limited in the present interaction, and it may well be that the statistical link between the incoming boundary layer, separated flow region and reflected shock wave is weaker here than in the other studies because the present interaction is only intermittently separated, and this may change the sensitivity of the shock wave to incoming disturbances. Comparisons between separated and intermittently separated interactions should therefore be made with care, although the conceptual model by Dupont et al. (2008) above is qualitatively consistent with the present study.

Finally, it may also be of interest to note that in the present study the joint p.d.f. shows a stronger correlation between the incoming boundary layer and reflected shock wave position than the incoming boundary layer and separated flow extent. This seemingly contradictory result can be explained because only the largest value of $\Delta$ was chosen within each realization, which exacerbates the correlation for small $\Delta$, since smaller values have been shown to have the largest spatial variation.

\subsection{The POD analysis}

To complete our discussion, we now decompose the fluctuating part of the flow field using the POD. Since the POD is based on ensemble-averaged correlations, 


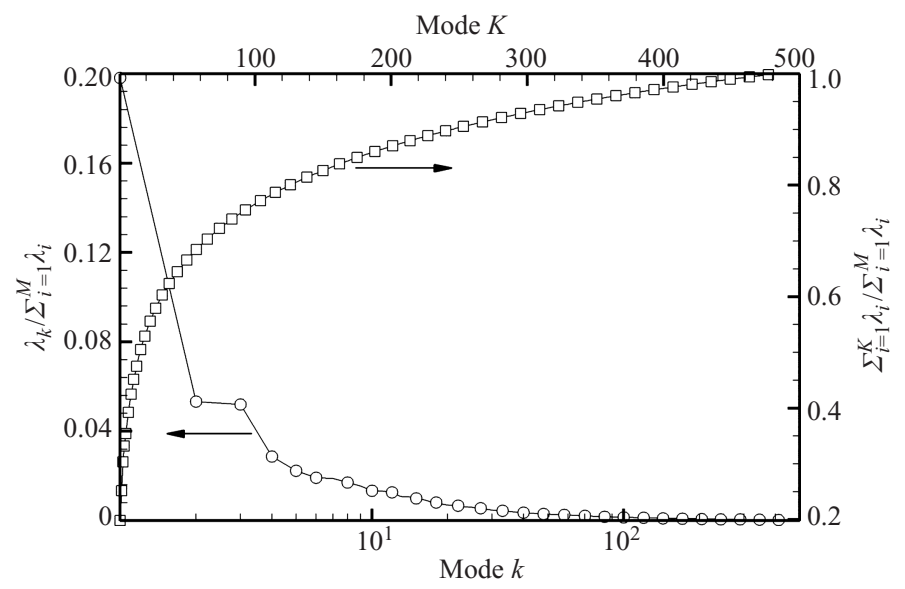

FIGURE 9. Eigenspectrum of the POD analysis. Modal energy content shown for the $k$ th eigenmode and cumulative modal energy shown for the $K$ th cumulative sum. Arrows assign data to the appropriate axes.

the eigenmodes represent only statistical information about the flow features they represent. A conceptually appealing interpretation is to view the following eigenmodes as perturbations to the mean flow field.

As the motivating prelude, figure 9 shows the modal energy and cumulative modal energy distributions of the POD eigenmodes. The POD eigenspectrum reveals that the first eigenmode is by far the most dominant, capturing almost $20 \%$ of the total energy. A mode that contributes a substantial proportion of the total energy of the flow is considered to play a more significant role in the flow dynamics than a mode that contains less energy (Kostas, Soria \& Chong 2005). It therefore appears that the first eigenmode represents an important contribution to the interaction's unsteadiness. Furthermore, Berkooz et al. (1993) have shown that if the eigenspectrum decays fast enough, then all the likely realizations can be found within a relatively small subset of eigenmodes. The cumulative ratio $\sum_{i=1}^{K} \lambda_{i} / \sum_{i=1}^{M} \lambda_{i}$ gives an indication of this energy convergence, by showing the amount of energy contained in the $M$ element system, contained in the first $K$ eigenmodes. To capture most of the energy, it is necessary that $\sum_{i=1}^{K} \lambda_{i} \approx \sum_{i=1}^{M} \lambda_{i}$ (and desirable that $K \ll M$ ). The cumulative energy distribution shows that over 70 modes are required to capture $75 \%$ (say) of the total energy. In comparison, other POD studies that have used planar PIV data, such as the one by Bernero \& Fiedler (2000), have reported about 20 modes in their study of a jet in counter-flow, and Patte-Rouland et al. (2001) have reported about 60 modes in their study of an annular jet, for the same amount of energy. Only a limited number of modes (say $<10$ ) contain an appreciable fraction of the total energy (say $>2 \%$ ), and we therefore restrict our attention to discussing them.

For the purposes of visualizing the two-dimensional eigenmodes, we first create a one-dimensional phase space $\phi$ using the temporal coefficients from the analysis. Recall that one can arbitrarily choose a finite number $K$ of the most energetic modes to form a subspace spanned by the first $K$ eigenmodes. Similarly, subspaces can be formulated based on a single eigenmode, by first ordering the temporal coefficients of all $M$ observations, such that $\phi_{n}^{k}=\left\{a_{k}\left(t_{n}\right) \leqslant a_{k}\left(t_{n+1}\right) \leqslant \ldots \leqslant a_{k}\left(t_{M}\right)\right\}$. An eigenmode then yields $M$ subspaces, the $n$th subspace of the $k$ th eigenmode $u_{n}^{k}(x, y)$ given by

$$
u_{n}^{k}(x, y)=a_{k}\left(t_{n}\right) \psi_{k}(x, y), \quad n=1, \ldots, M
$$


These subspaces provide a convenient method to analyse the behaviour of the $k$ th eigenmode. For notational convenience, we first define $\phi$ as

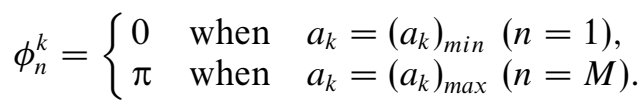

Using the above procedure, perspective renderings of the first four eigenmodes are shown in figure 10, sorted by decreasing fractional energy, with their modal number and relative energy content shown in the inset. The streamwise and wall-normal velocity components are displayed on the left and right, respectively. These figures may be conceptualized by considering the eigenmode in the $x-y$ plane and viewing it in the phase space $\phi$ along the spanwise coordinate. Since the temporal coefficients associated with each eigenmode for a particular realization can be either positive or negative, only the relative changes of sign within an eigenmode are important.

Recall that the first eigenmode is by far the most energetic. Figure 10(a) shows that this mode contains relatively large streamwise velocity fluctuations within the incoming boundary layer relatively close to the wall and separated flow region, in combination with wall-normal velocity fluctuations across the reflected shock wave, as shown in figure 10(b). (The curious reader may look ahead to figure 11 to see how the projection of this eigenmode on to the mean flow field contributes to the interaction's unsteadiness.) Note also the lack of velocity fluctuations along the incident shock wave, indicating that it is a steady feature on this energetic basis.

Higher-order eigenmodes have an energy level that is significantly less than the first eigenmode and portray a more intricate structure. The streamwise component of modes 2 and 3 depicted in figure $10(c, e)$, respectively, show that qualitative changes take place within the separation bubble and farther downstream, leading to an increase in the number of spatially extended regions, in the form of smaller-scale coherent flow features, often of opposite sign. The term 'subspace bifurcation' refers here to these qualitative changes. Such bifurcations are attributed to the higher-order harmonics required to properly represent the high-dimensional phase space of the data, which when used in combination with other modes would separate the different time scales within the interaction.

For instance, Dussauge et al. (2006) have compared energetic frequencies within the reflected shock wave region and separation bubble using fluctuating wall-pressure measurements in their Mach 2.3 shock reflection experiments and have found that they are both different, with the separation bubble having energetic frequencies that are an order of magnitude larger than those found in the reflected shock wave region. The subspace bifurcations observed to occur within the separation bubble are certainly consistent with the higher-order harmonics that would be required to properly represent this dynamical behaviour. The reader may note that no such bifurcation occurs within the reflected shock wave region between the first two modes, which represent over $25 \%$ of the total energy.

In contrast, mode 4 in figure $10(g, h)$ shows energetic fluctuations exclusively within the redeveloping boundary layer, in the form of distinct regions of relatively large streamwise and wall-normal velocity fluctuations of alternating sign. This pattern is consistent with the circular regions of wall-normal velocity often observed in the instantaneous realizations and is indicative of a train of quasi-streamwise vortices in the form of a convected vortex street. It therefore appears that the subspace features contained in the eigenmodes can represent the phenomenology observed in the instantaneous realizations. 

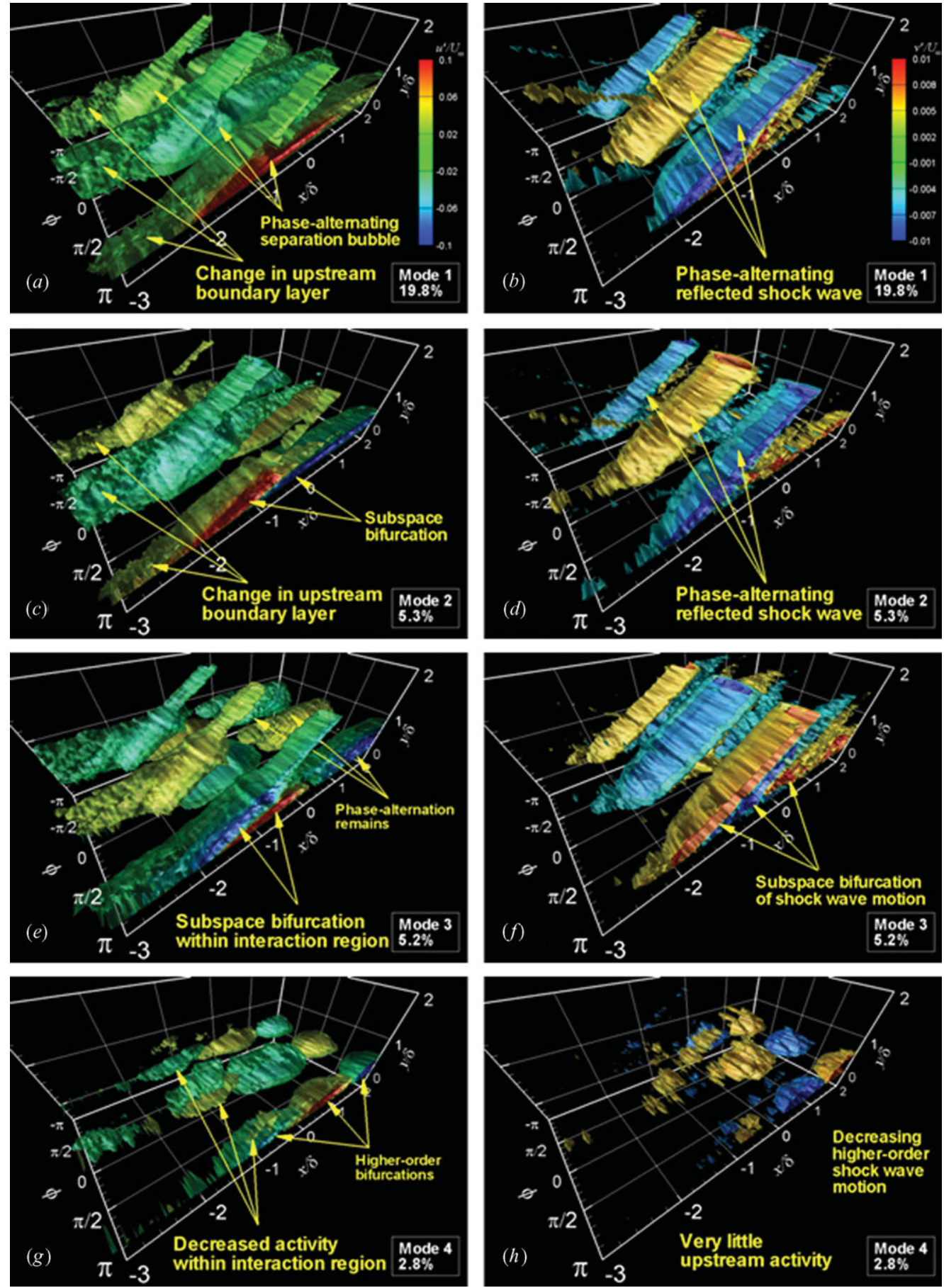

Figure 10. The POD eigenmodes: $u^{\prime} / U_{\infty}$ (left) and $v^{\prime} / U_{\infty}$ (right). (Note that modes $\geqslant 2$ show $2 u^{\prime} / U_{\infty}$.) Modal number and relative energy content are shown in the inset. 

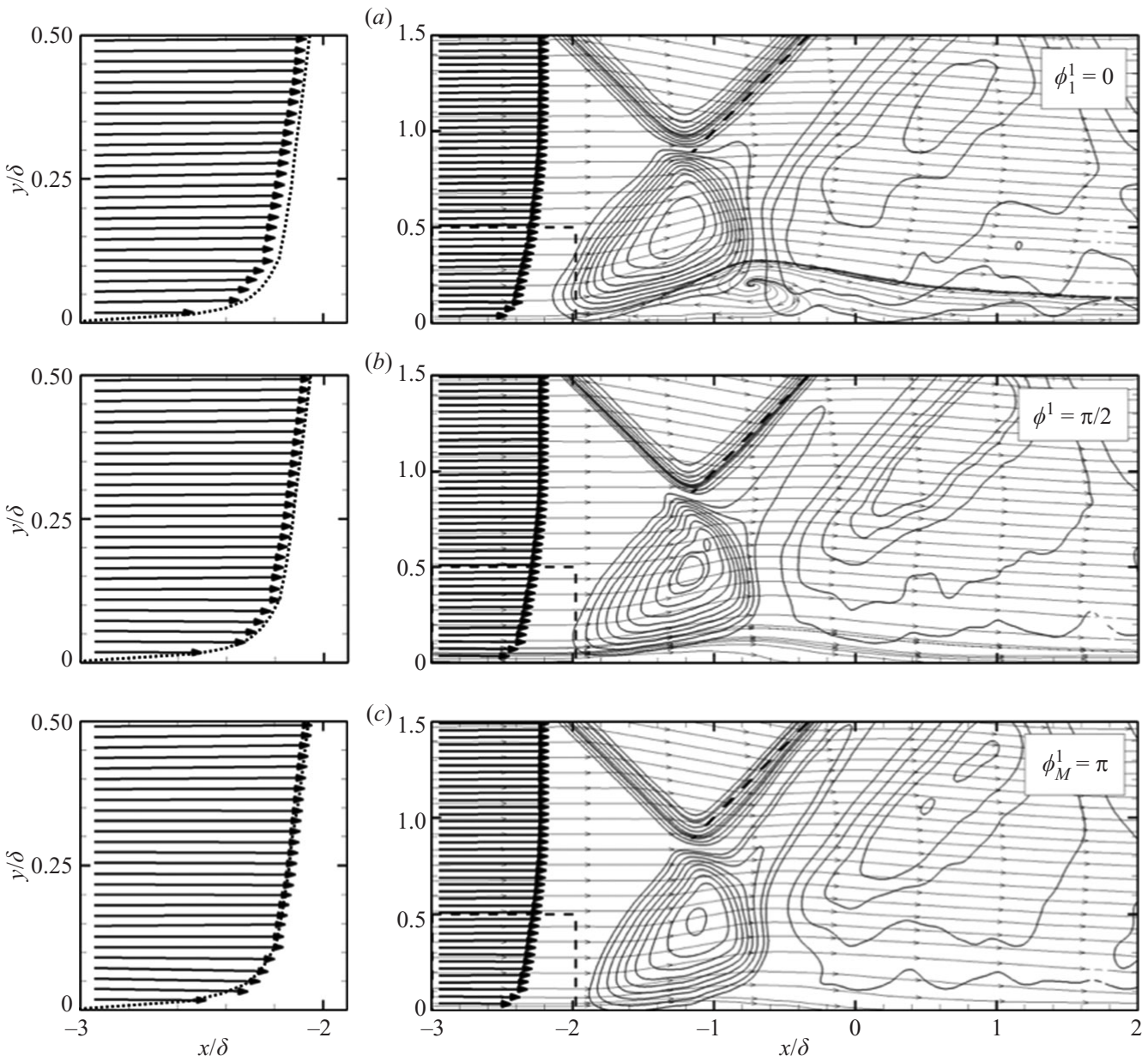

FIGURE 11. The first POD eigenmode superimposed on to mean flow at three different 'phases': (a) $\phi_{1}^{1}=0,(b) \phi^{1}=\pi / 2$ (mean flow field), (c) $\phi_{M}^{1}=\pi$. Wall-normal velocity contours are shown along with streamlines and incoming boundary layer velocity vectors. The mean position of the reflected shock wave is indicated by the dashed line. Shown on the left are zoomed velocity profiles of the incoming boundary layer for clarity. The dotted line indicates the mean velocity profile.

As an illustrative example to demonstrate the contribution of an eigenmode to the interaction's unsteadiness, the first eigenmode is superimposed on to the mean flow field. Now if the POD is considered to be a powerful method to analyse coherent behaviour in turbulent flows, then the direct analysis of the spatial modes in this way has to be carried out with care. This is because a major limitation is the loss of phase information, with velocity fluctuations within eigensubspace always in linear dependence with a temporal coefficient independent of time. Yet this procedure is both mathematically and conceptually permitted, since an instantaneous snapshot of the interaction may be represented by a combination of eigenmodes, each at an appropriate 'phase', as determined by the value of their respective temporal coefficient at that instant.

Figure 11 therefore shows the first eigenmode at three of these 'phases', namely $\phi_{1}^{1}=0, \phi^{1}=\pi / 2$ and $\phi_{M}^{1}=\pi$. The subspaces at $\phi_{1}^{1}=0$ and $\phi_{M}^{1}=\pi$ in figure $11(a, c)$, 
respectively, represent the contribution of the first eigenmode to the mean flow field when $a_{1}\left(t_{n}\right)$ is at its minimum and maximum, therefore representing the two extrema of the eignemode's influence as a perturbation to the mean flow field. The mean flow field itself occurs at $\phi^{1}=\pi / 2$, since $a_{1}\left(t_{n}\right)=0$, and is shown as the intermediate case in figure 11(b). Wall-normal velocity contours are also displayed with streamlines and incoming boundary layer velocity vectors. An indication of the mean position of the reflected shock wave is also shown by the dashed line. Zoomed incoming boundary layer profiles are displayed on the left for clarity. The dotted line indicates the mean velocity profile.

It can be seen that at $\phi_{1}^{1}=0$, a separation bubble is observed. The associated velocity vectors indicate that the incoming boundary layer is marginally less full than on the mean, and the reflected shock wave appears marginally farther upstream than on the mean. Conversely, at the other extreme when $\phi_{M}^{1}=\pi$, the flow remains fully attached. The associated velocity vectors indicate that the incoming boundary layer is marginally fuller than on the mean, and the reflected shock wave appears marginally farther downstream than on the mean. These observations are qualitatively consistent with the interrelationships portrayed in the joint p.d.f.s in figure 8. It should be remarked, however, that the quantitative effects of the first eigenmode on the mean flow field are surprisingly small, despite the eigenmode's relatively large fraction of the total energy. Indeed, attempts to rebuild the relationship between $1-1 / H_{i}$ and $\left(x-x_{\text {mean }}\right) / \delta$ using only the first eigenmode revealed that variations of $1-1 / H_{i}$ and $\left(x-x_{\text {mean }}\right) / \delta$ were less than $3 \%$ and $13 \%$ of the variations found in the original data, respectively. The interpretation of the first eigenmode (or any eigenmode) within the context of explaining the large-scale unsteadiness of the interaction must therefore be made with care, since it does not accurately describe the interaction's unsteadiness in any decisive way. This would necessitate the utility of additional eigenmodes in order to properly represent the interaction's unsteadiness. However, attempts at determining state-space relationships between the various temporal coefficients were unsuccessful, which substantiates the notion that the interaction's unsteadiness is a complex (high-dimensional) dynamical process.

\section{Summary and discussion}

We now consolidate the major observations made and formulate a simplified model of the interaction's unsteadiness, while at the same time making some judicious inferences to link it to what has been reported in the literature. In the model, the boundary layer first encounters the interaction, about two boundary layer thicknesses upstream of where the incident shock wave would impinge on the wall in the absence of the boundary layer. Here, incipient detachment occurs, and the probability of observing reversed flow rapidly increases farther downstream towards a maximal probability of about $50 \%$. The interaction in general may be qualitatively decomposed, on an instantaneous basis, into two layers, characterized by a highvelocity outer layer (typically $u / U_{\infty}>0.5$ ) and a low-velocity inner layer (typically $\left.u / U_{\infty}<0.5\right)$. These two layers are separated by a thin region of relatively high shear, in which discrete vortical structures are present.

These vortical structures are initially present close to the wall. Their initial distribution may be reconciled with the interaction's large-scale unsteadiness by drawing on the work of Kim \& Adrian (1999) and Adrian et al. (2000), who formulated a conceptual model consisting of the streamwise alignment of hairpin vortex structures into groups to form the so-called hairpin packets, and these packets 
then align to form very large-scale (i.e. $>3 \delta$ ) streamwise-elongated regions of relatively low- and high-speed fluid due to their induced motion. Although the present vortical structures appear as the cross-sectional view of a more complicated three-dimensional flow field, Humble et al. (2009) have recently investigated the present interaction's three-dimensional instantaneous structure using tomographic PIV and found evidence of a streamwise alignment of three-dimensional vortical structures within the incoming boundary layer, consistent with the hairpin-packet paradigm.

Such alignment gives rise to streamwise-elongated regions of relatively low- and high-speed fluid. In the present study, measurements made in the streamwise-wallnormal cross-sectional plane detect such regions as a sustained deformation of the instantaneous velocity profile, leading to a change in its fullness. One can now see how such small-scale structures could be associated with the large-scale unsteadiness reported here. This idea has also been reported by Ganapathisubramani et al. (2007) in their investigation of a compression ramp interaction; they also drew on the hairpin-packet model. In particular, they reported that such structures can extend up to $40 \delta$ in their Mach 2 boundary layer using Taylor's hypothesis, which they state could help account for the large-scale low-frequency unsteadiness.

As the interaction is approached, the vorticity layer and vortical structures are lifted away from the wall when flow separation is present. The structures ride over the separation bubble, treating it essentially like a streamlined obstacle, and create a fluid exchange between the outer and inner layers of the interaction. Outer high-velocity fluid is drawn in and ingested between the vortical structures, whereas low-velocity fluid from the separated flow region is transported away from the wall. As a result, the instantaneous reversed flow is intermittently supplied with fluid by the vortical structures as they propagate through the interaction and not from downstream as in the traditional (steady) view of separation, consistent with what is observed in incompressible separated flows. Even without a significantly reversed-flow region, a similar lifting of the vorticity layer and vortical structures can still occur due to the bulk dilatation of the inner (downstream moving) subsonic fluid.

The above behaviour may also be reconciled with the large-scale unsteadiness. Wu \& Martin (2008) have recently hypothesized that one of the mechanisms of the large-scale low-frequency motion of the shock wave involves a feedback loop between the separation bubble, separated shear layer and shock system. This mechanism involves large-scale enlarging and shrinking of the bubble that are associated with an instantaneous imbalance between shear layer entrainment from the separation bubble and fluid injection near reattachment and draws upon the earlier work of Eaton \& Johnston (1982), who carried out experiments in incompressible separated flows involving backward-facing steps. Kiya \& Sasaki (1985) further inferred from the model of Eaton \& Johnston (1982) that this imbalance is associated with the spatial organization of vortical structures, which play an important role in the instantaneous reattachment length by influencing the level of entrainment through a change in spanwise coherency. Within the context of the present study, one can envisage the passage of the present vortical structures, as they ride over the separation bubble, being associated with this spanwise coherency, with the resulting fluctuating level of momentum playing a role in the imbalance between shear layer entrainment from the separation bubble and fluid injection near reattachment and therefore the instantaneous bubble size. It is unclear at this point, however, how this mechanism may be reconciled with the influence of the vortical structures within the incoming boundary layer, but it is not inconceivable to believe that several mechanisms associated with the large-scale unsteadiness coexist. 


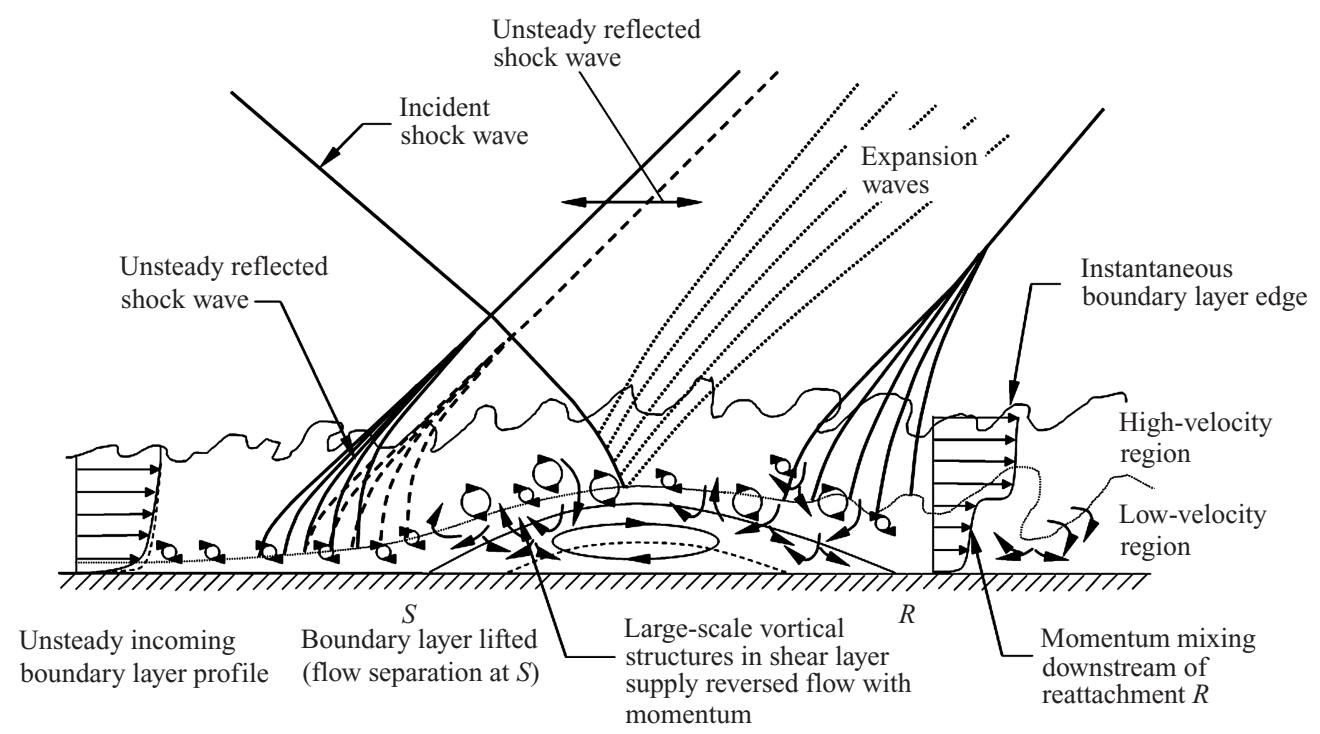

FIGURE 12. Conceptual model of the interaction's unsteadiness. The unsteady incoming boundary layer velocity profile is shown along with its association with the size of the separated flow region and reflected shock wave position. Also shown is the distinction between the inner and outer layers of the interaction, with discrete vortical structures along their interface.

Farther downstream, after turning around the separation bubble, the vortical structures often return to the proximity of the wall, whereas in other cases they become more broadly distributed normal to the wall than in the first part of the interaction. This behaviour is associated with the separation bubble's vortex-shedding process. While boundary layer recovery initiates downstream, complete recovery is not observed within the present measurement domain. A schematic of the model portraying the main phenomenological features discussed is shown in figure 12 .

\section{Conclusions}

Measurements of an incident SWTBLI at Mach 2.1 have been made using PIV in combination with data processing using the POD to obtain an instantaneous and statistical description of the unsteady flow organization. As a result, several conclusions may be drawn.

On an instantaneous basis, the interaction exhibits a multi-layered structure, characterized by a high-velocity outer region and a low-velocity inner region. The two regions are separated by a thin region of relatively high shear, which has an irregular and intermittent nature. Discrete vortical structures are prevalent along this interface, which appear to create an intermittent fluid exchange as they propagate downstream.

A statistical analysis confirms the existence of an intermittent separated flow region, with a streamwise extent within the range $0-2 \delta$. While flow reversal occurs in around half of the instantaneous realizations, the flow on average remains fully attached. The probability density rapidly decreases for an increasing bubble size, and no bubble sizes larger than $2 \delta$ are observed. There also exists momentarily small-scale flow reversal scattered both upstream and downstream of the interaction, with reversedflow regions occurring more frequently within the redeveloping boundary layer than within the incoming boundary layer. 
An interrelationship appears to exist between the fullness of the incoming boundary layer velocity profile, separated flow region and reflected shock wave. Joint p.d.f.s suggest that when $1-1 / H_{i}$ is greater than its median value (boundary layer less full), the streamwise extent of the (largest) separated flow region is more likely to be larger than its median size, and the reflected shock wave is more likely to be located downstream of its mean position, and vice versa. This relationship is weak, however, and this is thought to be associated with the intermittent nature of the interaction.

The eigenmodes returned from the POD show an energetic association between velocity fluctuations within the incoming boundary layer, separated flow region and across the reflected shock wave, and portray subspace features that represent, to a certain extent, the phenomenology observed in the instantaneous realizations. No single eigenmode, however, may be used to describe the interaction's unsteadiness in any decisive way, and the utility of higher-order eigenmodes is required to properly represent the complex dynamics of the interaction.

This work is supported by the Dutch Technology Foundation STW under the VIDI-Innovation Impulse program, grant DLR.6198. The authors would like to thank the reviewers for their helpful suggestions in the preparation of this paper.

\section{REFERENCES}

Adrian, R. J., Meinhart, C. D. \& Tomkins, C. D. 2000 Vortex organization in the outer region of the turbulent boundary layer. J. Fluid Mech. 442, 1-54.

Andreopoulos, Y. \& Muck, K. C. 1987 Some new aspects of the shock-wave/boundary-layer interaction in compression flows. J. Fluid Mech. 180, 405-428.

Aubry, N., Holmes, P., Lumley, J. P. \& Stone, E. 1988 The dynamics of coherent structures in the wall region of the turbulent boundary layer. J. Fluid Mech. 192, 115-173.

Beresh, S. J., Clemens, N. T. \& Dolling, D. S. 2002 Relationship between upstream turbulent boundary-layer velocity fluctuations and separation shock unsteadiness. AIAA J. 40, 24122422.

Berkooz, G., Holmes, P. \& Lumley, J. L. 1993 The proper orthogonal decomposition in the analysis of turbulent flows. Annu. Rev. Fluid Mech. 25, 539-575.

Bernero, S. \& Fiedler, H. E. 2000 Application of particle image velocimetry and proper orthogonal decomposition to the study of a jet in counterflow. Exp. Fluids 29, 274-281.

Bookey, P., Wyckham, C. \& Smits, A. J. 2005 Experimental investigations of Mach 3 shock-wave turbulent boundary layer interactions. Paper 2005-4899. AIAA.

Chong, M. S., Soria, J., Perry, A. E., Chacin, J., Cantwell, B. J. \& Na, Y. 1998 Turbulence structures of wall-bounded shear flows using DNS data. J. Fluid Mech. 357, 225-247.

Délery, J. \& MARvin J. G. 1986 Shock-wave boundary layer interactions. AGARDograph 280. AGARD.

Dolling, D. S. 2001 Fifty years of shock wave/boundary layer interaction research: what next? AIAA J. 39, 1517-1531.

Dolling, D. S. \& Murphy, M. T. 1983 Unsteadiness of the separation shock wave structure in a supersonic compression ramp flow-field. AIAA J. 21, 1628-1634.

Dupont, P., Haddad, C. \& Debiève, J. F. 2006 Space and time organization in a shock-induced separated boundary layer. J. Fluid Mech. 559, 255-277.

Dupont, P., Piponniau, S., Sidorenko, A. \& Debiève, J. F. 2008 Investigation by particle image velocimetry measurements of oblique shock reflection with separation. AIAA J. 46, 1365-1370.

Dussauge, J.-P., Dupont, P. \& Debiève, J. F. 2006 Unsteadiness in shock wave boundary layer interactions with separation. Aerosp. Sci. Technol. 10, 85-91.

Eaton, J. K. \& Johnston, J. P. 1982 Low-frequency unsteadiness of a reattaching turbulent shear layer. In Turbulent Shear Flows 3 (ed. L. J. S. Bradbury, F. Durst, B. E. Launder, F. W. Schmidt \& J. H. Whitelaw), pp. 162-170. Springer. 
Elena, M. \& Lacharme, J. P. 1988 Experimental study of a supersonic turbulent boundary layer using a laser Doppler anemometer. J. Theoret. Appl. Mech. 7, 175-190.

Erengil, M. E. \& Dolling, D. S. 1993 Physical causes of separation shock unsteadiness in shock wave/turbulent boundary layer interactions. Paper 93-3134. AIAA.

Ganapathisubramani, B., Clemens, N. T. \& Dolling, D. S. 2006 Large-scale motions in a supersonic turbulent boundary layer. J. Fluid Mech. 556, 271-282.

Ganapathisubramani, B., Clemens, N. T. \& Dolling, D. S. 2007 Effects of upstream boundary layer on the unsteadiness of shock-induced separation. J. Fluid Mech. 585, 369-394.

Hou, Y. X. 2003 Particle image velocimetry study of shock-induced turbulent boundary layer separation. PhD dissertation, Department of Aerospace Engineering and Engineering Mechanics, University of Texas at Austin.

Humble, R. A., Elsinga, G. E., Scarano, F. \& van Oudheusden, B. W. 2009 Three-dimensional instantaneous structure of a shock wave/turbulent boundary layer interaction. J. Fluid Mech. 622, 33-62.

Humble, R. A., Scarano, F. \& van Oudheusden, B. W. 2007 Particle image velocimetry measurements of a shock wave/turbulent boundary layer interaction. Exp. Fluids 43, 173-183.

Hunt, J. C. R., Wray, A. A. \& Moin, P. 1988 Eddies, streams, and convergence zones in turbulent flows. Report CTR-S88. Center for Turbulence Research.

JeOng, J., Hussain, F., Schoppa, W. \& KIM, J. 1997 Coherent structures near the wall in a turbulent channel flow. J. Fluid Mech. 332, 185-214.

Kim, K. C. \& Adrian, R. J. 1999 Very large-scale motion in the outer layer. Phys. Fluids 11, $417-422$.

KIYA, M. \& SASAKI, K. 1985 Structure of large-scale vortices and unsteady reverse flow in the reattaching zone of a turbulent separation bubble. J. Fluid Mech. 154, 463-491.

Kostas, J., Soria, J. \& Chong, M. S. 2005 A comparison between snapshot POD analysis of PIV velocity and vorticity data. Exp. Fluids 38, 146-160.

Lumley, J. L. 1967 The structure of inhomogeneous turbulence. In Atmospheric Turbulence and Wave Propagation (ed. A. M. Yaglom \& V. I. Tatarski), pp. 166-178. Nauka.

Moreno, D., Krothapalli, A., Alkislar, M. B. \& Lourenco, L. M. 2004 Low-dimensional model of a supersonic rectangular jet. Phys. Rev. E 69, 026304.

NA, Y. \& MorN, P. 1998 Direct numerical simulation of a separated turbulent boundary layer. J. Fluid Mech. 374, 379-405.

Patte-Rouland, B., Lalizel, G., Moreau, J. \& Rouland, E. 2001 Flow analysis of an annular jet by particle image velocimetry and proper orthogonal decomposition. Meas. Sci. Technol. 12, $1404-1412$.

Pirozzoli, S. \& Grasso, F. 2006 Direct numerical simulation of impinging shock wave/turbulent boundary layer interaction at $M=2.25$. Phys. Fluids 18, 065113.

Ringuette, M. J., Wu, M. \& Martin, M. P. 2008 Coherent structures in direct numerical simulation of turbulent boundary layers at Mach 3. J. Fluid Mech. 594, 59-69.

Rowley, C. W., Colonius, T. \& Murray, R. M. 2004 Model reduction for compressible flows using POD and Galerkin projection. Physica D 189, 115-129.

SAmimy, M. \& Lele, S. K. 1991 Motion of particles with inertia in a compressible free shear layer. Phys. Fluids 3, 1915-1923.

Scarano, F. \& Riethmuller, M. L. 2000 Advances in iterative multi-grid PIV processing. Exp. Fluids 29, S051.

Schrijer, F. F. J., Scarano, F. \& van Oudheusden, B. W. 2006 Application of PIV in a Mach 7 double-ramp flow. Exp. Fluids 41, 353-363.

Simpson, R. L. 1989 Turbulent boundary-layer separation. Annu. Rev. Fluid Mech. 21, 205-234.

Sirovich, L. 1987 Turbulence and the dynamics of coherent structures. Quart. Appl. Math. 45, 561-571.

Smith, M. W. \& Smits, A. J. 1995 Visualization of the structure of supersonic turbulent boundary layers. Exp. Fluids 18, 288-302.

Westerweel, J. \& Scarano, F. 2005 Universal outlier detection for PIV data. Exp. Fluids 39, 1096-1100.

Wu, M. \& Martin, M. P. 2008 Analysis of shock motion in shockwave and turbulent boundary layer interaction using direct numerical simulation data. J. Fluid Mech. 594, 71-83. 